\title{
SYNTHESIS OF SILVER NANOPARTICLES ON A SOLID SURFACE USING A CLASSICAL PHOTOGRAPHIC METHOD
}

By

\author{
Saif Al-Alul \\ B.Sc., Ryerson University, 2011
}

\author{
A thesis \\ presented to Ryerson University \\ in partial fulfillment of the \\ requirements for the degree of \\ Master of Science \\ in the program of \\ Molecular science
}

Toronto, Ontario, Canada, 2014

CSaif Al-Alul 2014 


\section{Author's Declaration}

I hereby declare that I am the sole author of this thesis. This is a true copy of the thesis, including any required final revisions, as accepted by my examiners.

I authorize Ryerson University to lend this thesis to other institutions or individuals for the purpose of scholarly research

I further authorize Ryerson University to reproduce this thesis by photocopying or by other means, in total or in part, at the request of other institutions or individuals for the purpose of scholarly research.

I understand that my thesis may be made electronically available to the public 


\title{
SYNTHESIS OF SILVER NANOPARTICLES ON A SOLID SURFACE USING A CLASSICAL PHOTOGRAPHIC METHOD
}

\author{
Saif Al-Alul \\ Master of Science, Molecular Science, Ryerson University 2014
}

\begin{abstract}
A classical photographic method, the Becquerel method, produces a positive image comprised of silver nanoparticles on a silver surface. The particles are grown by exposing an iodised silver plate to light in the blue or ultraviolet, which initiates the formation of particles, followed by development (growth) with light in the red portion of the spectrum. Because the Becquerel method is essentially a means of producing a surface of patterned nanoparticles, it also has potential technological applications. This thesis is a systematic investigation of the Becquerel method. . It was determined that the initiation of nanoparticles is effective for wavelengths in the range 447 to $254 \mathrm{~nm}$. The sudden rise in nanoparticle production around $447 \mathrm{~nm}$ implicates direct excitation of the AgI layer in the initiation step; however, the behaviour of the action spectrum at shorter wavelengths implies an electron-mediated mechanism. It is possible that both direct excitation and electron-mediated processes occur in the ultraviolet. Scanning electron micrographs indicate that nanoparticle morphology may be dependent on the initiation wavelength, with longer wavelengths producing a variety of shapes, while shorter wavelengths produce primarily dots. Nanoparticle growth (development) was achieved with all wavelengths studied; however, shorter wavelengths photons were more effective than longer wavelengths. The results from a study of the aging of the AgI film suggested that there is period of one or two days during which the film 'matures,' becoming more effective for nanoparticle production.
\end{abstract}




\section{Acknowledgement}

First and foremost, I would like to extend my deepest gratitude to Dr.Darrick Heyd for the research opportunity to explore such a novel and intriguing project. None of this would have been possible if it was not for his endless support, guidance, and encouragement. Everything I have learned from him is truly priceless.

I would also like to thank Mike Robinson, for his guidance and aid with understanding the Becquerel process of photography. Little is documented on the Becquerel technique. His experience and aid made this project possible.

A thanks is also extended to undergraduate student, Sukhvinder Bharaj. In addition to aiding me with much of the lab work and research, he was also great friend. His company will be missed.

I also would like to thank technologists Shawn McFadden, Sylvia O’Sullivan, and Wei Zhang for all their help.

In addition, I would like to thank a number of students who have never hesitated to help. This includes Eric Da Silva, Jesse Quinn, Rob Denning, Jonathan Ward, Devin Machin and Ali Naqvi. All their help was greatly appreciated.

Also, thanks to Qiang Li, for all the SEM data.

Finally, I would like to thank my father, Mazooz Al-Alul, my mother, Noura Osman, my brother, Yazan Al-Alul, and my sister, Loujain Al-Alul, for all their patience and moral support. I could not have made it through all this without them. 


\section{Table of Contents}

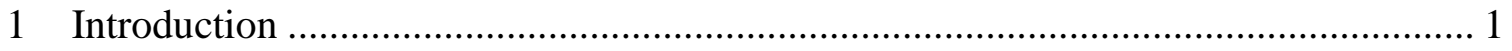

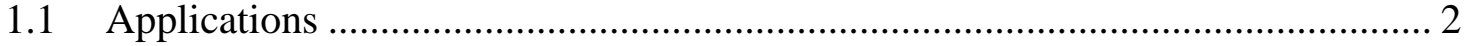

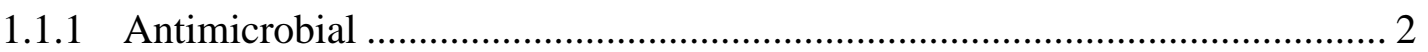

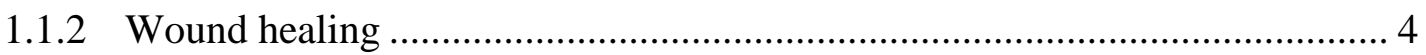

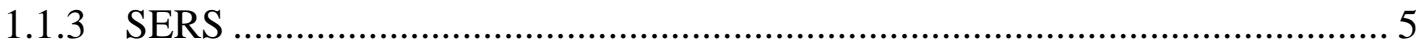

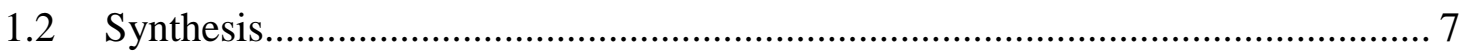

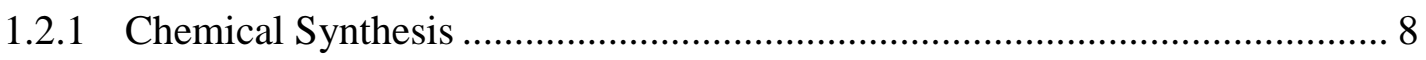

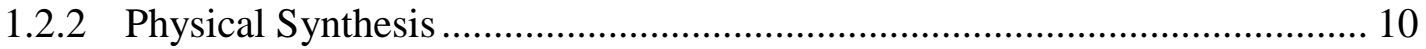

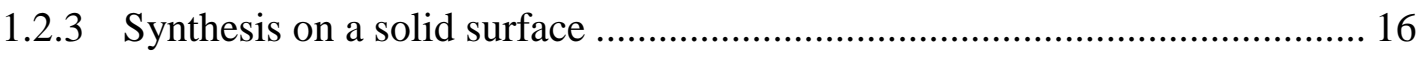

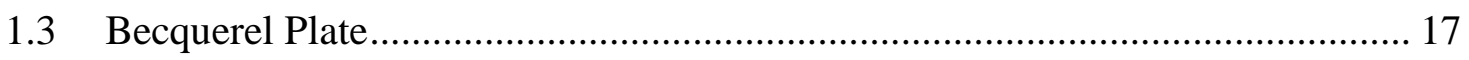

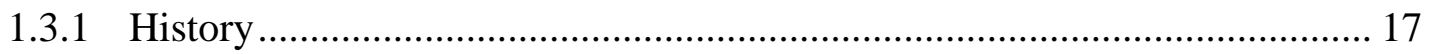

1.3.2 Studying the Becquerel method for nanoparticle synthesis ......................... 19

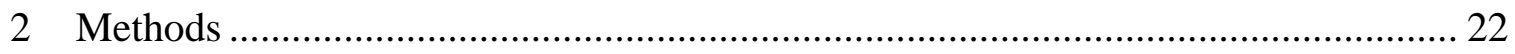

$2.1 \quad$ Specialized equipment.................................................................................. 22

2.1.1 Iodine Chamber .................................................................................. 22

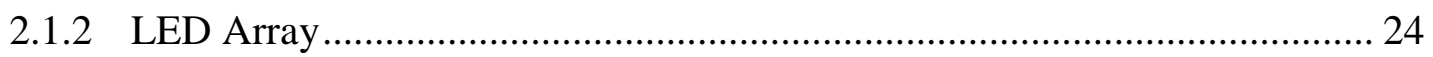

2.2 Plate Preparation ........................................................................................ 26

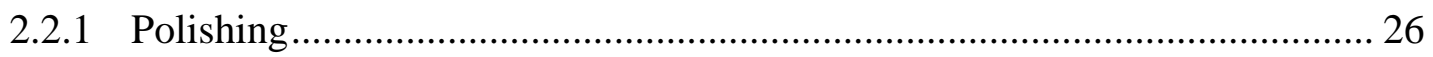

2.2.2 Iodization of the silver plate ................................................................... 26

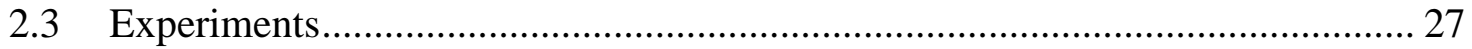

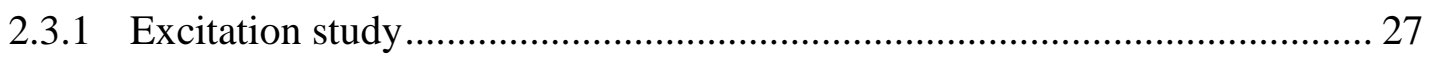

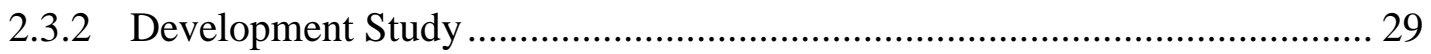

2.3.3 Iodine Maturation Study.......................................................................... 29

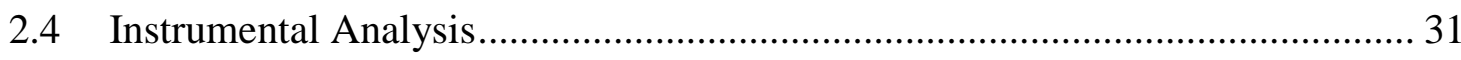

2.4.1 ICP-AES Analysis ................................................................................ 31

2.4.2 Scanning Electron Microscope (SEM) Analysis ............................................ 31 


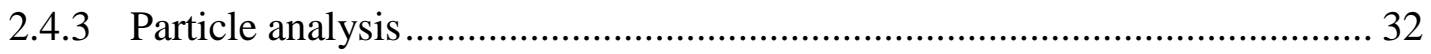

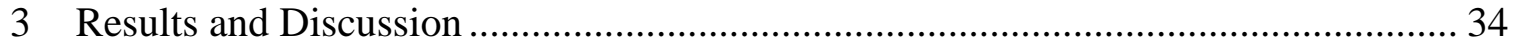

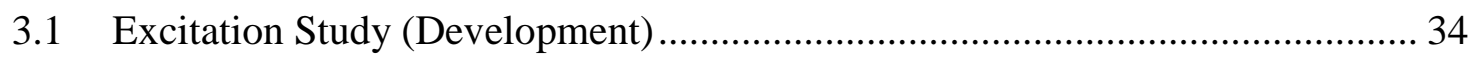

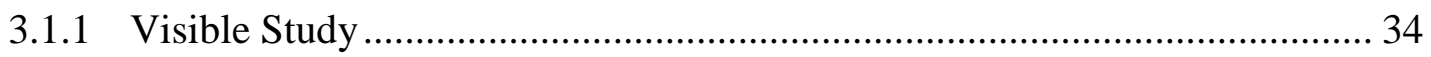

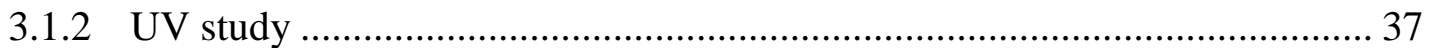

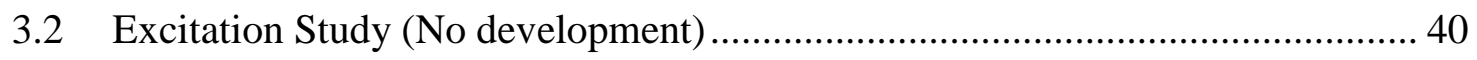

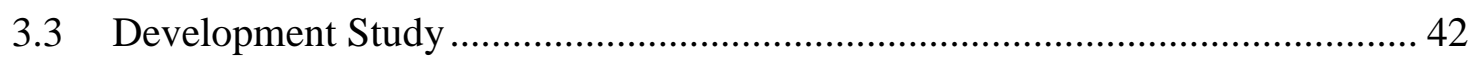

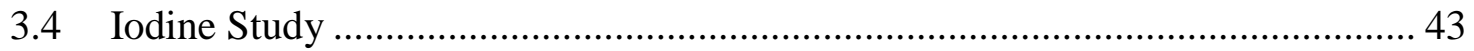

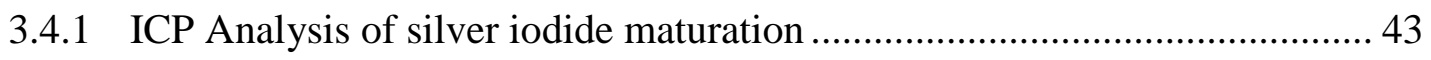

3.4.2 SEM analysis of silver iodide maturation .................................................. 45

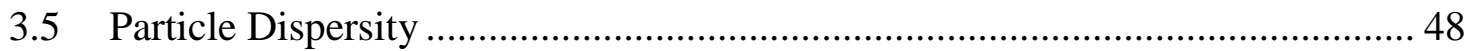

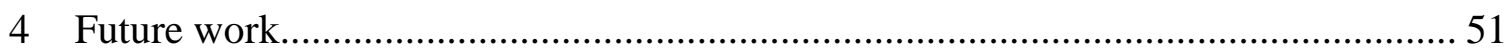

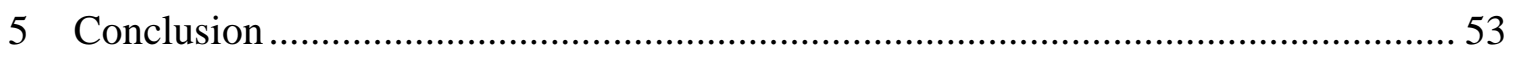




\section{Table of Figures}

Figure 2.1: Jacketed beaker for containing $I_{2}$ and controlling the temperature of its vapor....... 22

Figure 2.2: The plate holder. The top image is the top view, the bottom is a bottom view....... 23

Figure 2.3: The adaptor that connects the plate holder and the iodine chamber together. Top image shows the top view, and bottom image shows the bottom view 23

Figure 2.4: A front view of the Thermopile holder. The centre vertical slit is where the thermopile is mounted, allowing for vertical translation. 24

Figure 2.5: LED array, with wavelengths of $447 \mathrm{~nm}, 470 \mathrm{~nm}, 505 \mathrm{~nm}, 530 \mathrm{~nm}, 591 \mathrm{~nm}, 617 \mathrm{~nm}$, $627 \mathrm{~nm}$, and $655 \mathrm{~nm}$. The top is the front view, and the bottom is the rear view. 25

Figure 3.1: A graph of the number of particles produced by each LED during the excitation

study. 34

Figure 3.2: Particles per photon (solid dots, solid lines) for wavelengths 406-nm through to 254nm compared to imaginary refractive index of silver metal (hollow dots, dashed lines)

Figure 3.3: SEM images of particles produced with 302nm (top) and 334nm (bottom) 40

Figure 3.4: Top image was exposed for 10 seconds with $447 \mathrm{~nm}$ and developed with $655 \mathrm{~nm}$ light for 10 minutes. Bottom image was exposed with $447 \mathrm{~nm}$ for 4 minutes 41 
Figure 3.5: Number of particles produced with for wavelengths $447 \mathrm{~nm}, 470 \mathrm{~nm}, 505 \mathrm{~nm}$ and $530 \mathrm{~nm}$. No $655 \mathrm{~nm}$ light development was used

Figure 3.6: Number of particles produced when developed with $505 \mathrm{~nm}, 530 \mathrm{~nm}, 591 \mathrm{~nm} 617 \mathrm{~nm}$

$627 \mathrm{~nm}$ and $655 \mathrm{~nm}$

Figure 3.7: Average particle size produced by development with $505 \mathrm{~nm}, 530 \mathrm{~nm}, 591 \mathrm{~nm}$, $617 \mathrm{~nm}, 627 \mathrm{~nm}$, and $655 \mathrm{~nm}$. 43

Figure 3.8: Maturation study of the silver iodide. 44

Figure 3.9: Maturation of the iodide layer. Changes in the iodide layer during the first day after iodine exposure result in enhanced particle formation 47

Figure 3.10: Histogram of 447nm LED exposure, developed with 655nm LED. 48

Figure 3.11: PDI value for the developed excitation study for both UV and visible. 49

Figure 3.12: PDI value for excitation without development. 49 


\section{Table of Abbreviations}

$\begin{array}{ll}\text { AFM } & \text { Atomic force microscope } \\ \text { AgNP } & \text { Silver nanoparticles } \\ \text { BVPP } & \text { 1,4-bis[2-(4- pyridyl)ethenyl]-benzene } \\ \text { LED } & \text { Light emitting diode } \\ \text { LSRP } & \text { Localized surface plasmon resonance } \\ \text { PDDA } & \text { of poly(diallydimethylammonium chloride) } \\ \text { PDI } & \text { Polydispersity index } \\ \text { PEG } & \text { Polyethyleneglycol } \\ \text { PVA } & \text { Polyvinylalcohol } \\ \text { PVP } & \text { Polyvinylpyrrolidone } \\ \text { SEM } & \text { Scanning electron microscope } \\ \text { SERS } & \text { Surface enhanced microscope } \\ \text { TEM } & \text { Transmission electron microscope } \\ \text { VFM } & \text { Variable frequency microwave }\end{array}$




\section{Introduction}

Silver nanoparticles are known to have antimicrobial, wound healing, and catalytic properties. Although silver metal is catalytic, silver nanoparticles (AgNP) have enhanced catalytic properties, with a wider range of reactions possible, including formation of ethylene, propylene oxide and formaldehyde.

Nanoparticles are usually defined as particles that have at least one dimension that is between $1 \mathrm{~nm}$ and $100 \mathrm{~nm}$ in length. ${ }^{1}$ The geometry of the particle is irrelevant to its definition. The small sizes of the particles provide an increase in the surface to volume ratio. This increase in the surface to volume ratio results in an increase in surface energy. ${ }^{2}$ In addition, the size of the particles has an effect on the electronic structure of the particles. The ability to control the size of the particles is crucial for the formation of these unique properties, which is significant for the desired application.

While the nanotechnology research has been underway for over thirty years, there is some evidence to suggest that the formation and use of nanoparticles may have been around for much longer than that, without referring to them with the term "nano"., There is evidence to suggest the use of nanoparticles by glass makers, unknowingly, during the $4^{\text {th }}$ century in the Roman Empire with the discovery of the Lycurgus Cup. ${ }^{4}$

The first publication of the synthesis of colloidal silver was in 1889 by M. C. Lea. ${ }^{5}$ Such colloids had many medical applications, but were primarily used as disinfectants. Since 1897 , medical products that use colloidal silver have been named "collargol". By 1954, "Algaedyn" became the registered name for that product. Prior to the 1980s, when the nanotechnology 
research began to boom, most research on AgNP was conducted with the sole purpose for use in surface enhanced Raman spectroscopy(SERS). ${ }^{4}$ Once additional properties of AgNP were observed, further studies were conducted for wider applications.

Many methods of synthesizing AgNP have been discovered, some of which provide a variety in characteristics of the particles, such as size, shape and protective agent. Most of these methods synthesize the particles in solution. One of the most significant applications of AgNP is use with SERS, which requires the particles to be present on a solid surface. More complex procedures are required in order to embed the particles on a solid surface after synthesis.

In this study, a classical photographic method of creating images on a silver plate, known as Becquerel photography, will be explored for use in creating AgNP. This method obviates the complex steps for embedding nanoparticles on a solid surface because particles are created in situ on the solid surface. The method also requires no solvents or adjuncts to create uncoated (bare) silver nanoparticles that can be patterned on a sub-micron scale.

\subsection{Applications}

\subsubsection{Antimicrobial}

The antimicrobial properties of bulk silver have been known for centuries. ${ }^{6}$ The earliest mention of its use can be traced back to Roman pharmacopoeia texts from 69B.C. This brought about the popularity of using silver containers, or silver lined containers for storing water. Ancient cultures in Mexico used silver containers to store water and milk. Its role as a disinfectant is still in use today; the MIR space station and NASA shuttles use silver to treat recycled water. For AgNP, the increase in surface area to volume ratio results in an enhancement 
in its antimicrobial properties. Such enhancement explains why silver nanoparticles receive tremendous attention from researchers.

While silver is capable of eliminating a wide variety of microbes, the mechanism of accomplishing this is not well understood. Numerous mechanisms have been proposed, which range from the binding and disruption of cellular membrane, to binding straight onto DNA and inhibiting reproduction. ${ }^{7}$ The mechanism does appear to be reliant on the microbe in question, regardless of the type of family that the virus may belong to. ${ }^{8}$

In their study, Parashar et al. have discussed two methods of synthesizing AgNP, a generic chemical method and a green method, using Guava leaf. ${ }^{7}$ The author then compared the effectiveness of the two synthetic varieties at killing $E$. coli and observed the progress using a transmission electron microscope (TEM) over a 12 hour period. After one hour, the green nanoparticles begin to interact with the E.coli, trying to adhere to the cellular membrane of E.coli. At 5 hours, the nanoparticles begin to anchor to the cellular membrane, and some of the particles had managed to make their way inside the cell. At 8 hours, the particles completely cover the E.coli cell, and the structural integrity of the cellular membrane begins to collapse, resulting in the death of the cell. Through the use of quantum chemical calculations, the authors theorize that the silver interacts with molecules that contain $\mathrm{C}-\mathrm{O}$ bonds, such as lipopolysaccharides and lysozymes. Once the silver comes into close proximity to a C-O bond, the bond begins to slightly stretch, weakening the bond. As more and more silver atoms begin to cluster around the $\mathrm{C}-\mathrm{O}$ bond, the effect of the silver on the bond magnifies, further weakening the bond. Once the bond breaks, the molecule will break down, which will eventually result in the collapse of the cellular membrane, followed by cell death. This theory also suggests why 
bulk silver may be less effective than nanoparticles as a disinfectant. Since nanoparticles are small enough to concentrates its efforts on to a single C-O bond, the effects will be magnified.

A review paper by Galdiero et al. discusses the antimicrobial properties of silver

nanoparticles, specifically with viruses. ${ }^{8}$ It is reported that for each virus type, the mechanism of action the silver undergoes in killing the virus is different. For example, silver interacts with Hepatitis B's DNA, inhibiting its ability to replicate in another host's cells. The ability of Influenza virus, monkeypox virus and herpes simplex virus type 1 to bind to a plasma membrane is inhibited by silver. And Tacaribe virus becomes inactive before entering the cell. With such a broad spectrum of microbes being targeting by a variety of mechanisms, it is unlikely that the viruses can develop resistance to silver.

\subsubsection{Wound healing}

In addition to eliminating microbes, silver has the ability to heal wounds more rapidly, compared to no treatment method. ${ }^{9}$ The treated wound would also have diminished or no scarring as opposed to the no treatment scenario, and sometimes the restoration of hair growth on the affected area. Tian et al. have studied the healing of a variety of wounds on rats when treated with silver nanoparticles and no treatment. With regards to thermal injury, a wound with no treatment was fully healed in 35 days. When treated with silver nanoparticles, the wound was healed in 25 days instead. When comparing the treated and untreated skin after complete healing, the treated skin had a closer resemblance to normal skin than the untreated, in addition to less scarring.

It is believed that the antimicrobial properties of AgNP may be one of the contributors to the enhanced healing. Tian et al. have determined that no bacteria would be present at the wound 
for at least 7 days when applying a AgNP treated bandage. In addition, AgNP has the ability to modulate the cytokines involvement in wound healing. This results in reduced inflammation, enhanced healing and skin regeneration, and a reduction in the formation of scars.

\subsubsection{SERS}

Of all the applications that exist for silver nanoparticles, SERS is probably the most studied. When using AgNP with Raman spectroscopy, the Raman signal can be enhanced many orders of magnitude, sometimes even high enough to permit single molecule detection. ${ }^{10}$ Due to such high sensitivity, SERS is often considered to have the ultimate limit of detection. Such high sensitivity makes SERS ideal for use in chemical analysis in a wide variety of fields, including polymer science, materials science, biosensing, catalysis and electrochemistry. ${ }^{11}$

SERS was first discovered in 1974 by Fleischmann. ${ }^{12,13}$ It was initially believed that the observed enhancement was caused by the roughness of the surface; the rougher the surface, the higher the surface area, hence more signal per area. Four years later, it was discovered that the enhancement was actually caused by localized surface plasmon resonance (LSPR). LSPR generates and enhances local electric fields, which creates small "hot spots" on to the surface of nanoparticles. Any molecules that are caught within these "hot spots" experience an enhancement in Raman scattering, which will significantly boost the signal. ${ }^{10}$ Coinage metals are usually used for SERS, since the LSPR occurs in visible and near IR.

The enhancement in the generated Raman signal is reliant on the interaction between the molecule of interest and the nanoparticles. Hence, the size, shape and morphology of the nanoparticles will have an impact on the signal. Having good control over the nanoparticle 
characteristics while synthesizing the AgNP is crucial if a high quality Raman signal is to be achieved.

A study by Jia et al. explored the method of synthesizing nanoparticles on a solid substrate using the layer by layer technique, in addition to the effect of particle size on Raman signal. Each layer was prepared by dipping the substrate into a solution of silver nitrate for a set duration, with 10 hours as the maximum duration. The longer the substrate remained in the solution, the larger the AgNP became. The Raman enhancement was measured at different durations of growth, using 1,4-bis[2-(4- pyridyl)ethenyl]-benzene (BVPP) as the probing molecule. It was observed that the Raman signal would get enhanced after longer durations of growth. After 1 hour of growth, $10 \mathrm{~nm}$ sized particles produced a very weak Raman signal. The strength of the Raman signal continued to grow as the particles grew in size. The signal strength reached a maximum at 8 hours of growth, with a particle size of $90 \mathrm{~nm}$. The signal then begins to degrade slowly after 8 hour growth, with particles having a size of $150 \mathrm{~nm}$ at 10 hours.

To obtain a SERS active system, the size of metal nanoparticles will have to be typically between 5 and $100 \mathrm{~nm}$ in size. ${ }^{13}$ When nanoparticles reach sizes that are larger than $100 \mathrm{~nm}$, excitation of dipole plasmons will no longer exclusively occur, and instead cause higher order multipoles to get excited. Excited multipoles are not radiative, and will produce no Raman enhancement. When the particles are too small, the conductivity of the metal nanoparticles is reduced due to electronic scattering process. This will result in a very weak Raman signal.

The stability of the particles was also studied, to determine how long after the synthesis process can the particles still be used for Raman enhancement. The stability was determined by measuring the Raman signal over a set period of time. After 50 days of storing the slide, the 
Raman signal degraded by $30 \%$. To observe a change in the Raman signal intensity, a change in the size, shape or morphology of the particles must occur. An atomic force microscopy (AFM) analysis was performed on the slides to determine of such a change has occurred. The size and morphology were unchanged, however the shape of the particles was affected. This shows how sensitive Raman enhancement is when it comes to such minute changes. Having good control of the particle's size, shape and morphology during synthesis is crucial to maximize the enhancement effect.

The effects of the shape of the nanoparticles on the Raman signal have also been studied. ${ }^{10}$ A comparison in E-field between a nanocube and a nanosphere was carried out. For the nanocube, the E-field was extremely weak at the edges of the cube. At the corners for the cube, however, the E-field is at its strongest. The nanosphere on the other hand had an equal and weak E-field strength all around. When comparing the E-field of the two shapes, the corners of the nanocube have an E-field 60X stronger than the nanosphere. This shows that the orientation of the nanoparticle is important to maximize Raman enhancement. A pair of nanocube particles with the corners next to one another was found to have a stronger signal than a corner to edge pair.

\subsection{Synthesis}

Many methods of synthesizing AgNP have been developed over the last 3 decades. Each method provides variations in the characteristics of nanoparticle production. These methods are usually placed under two general categories; chemical synthesis and physical synthesis. 


\subsubsection{Chemical Synthesis}

\subsubsection{Synthesis}

The synthesis of AgNP was first achieved through the use of a chemical method. This method was first discovered by M.C. Lea in 1889. Lea accomplished the synthesis by reducing silver citrate with ferrous citrate. At the time, Lea was unaware of the fact that he has formed silver particles that were on the order of nanometers in size, and referred to the product as allotropic silver. Since then, many methods of chemically synthesizing AgNP have been developed. Generally, most of these methods follow a similar methodology to Lea's: silver salts are reduced with the aid of a reducing agent. ${ }^{2}$ There are many variables that have been determined to affect AgNP characteristics, including concentration, reducing agent ${ }^{14}$, solution ${ }^{15}$, temperature ${ }^{16}$ and $\mathrm{pH}$.

Much research has been performed to determine the effect the reducing agent has on the formation of silver nanoparticles. Van Hoonacker et al. has conducted a study to compare the reducing agents citrate and sodium borohydride's reducing effects on silver nitrate. ${ }^{14}$ They showed that the strength of the reducing agent affects the size of the particles. Sodium borohydride, a strong reducing agent compared to citrate, yields small particles, averaging at about $10 \mathrm{~nm}$. Citrate on the other hand, produced particles at approximately $40 \mathrm{~nm}$ in size. By choosing the right reducing agent, nanoparticle size can be controlled.

The solution in which the particles are synthesized at also has an effect on the particle size. Jacob et al. have studied AgNP synthesis in glycol and water mixture. ${ }^{15}$ By varying the glycol to water ratio, different nanoparticle sizes were achieved. At 99:1, 25:75, and 1:99 ratios of glycol: water, $17 \mathrm{~nm}, 22 \mathrm{~nm}$, and 70nm size particles were obtained, respectively. Having 
control on the nanoparticle size without altering the source of the silver or the reducing agent is an advantageous feature, as it helps perfect the particles for the desired application

Velikov et al. have studied the effect of the reaction mixture's $\mathrm{pH}$ on particle size. At higher $\mathrm{pH}$ values, smaller particles are produced, while with lower $\mathrm{pH}$ results in larger particles. At high $\mathrm{pH}$, the nanoparticles will have a large surface charge which reduces the ionic strength, which results in a diminishment in aggregation. Hence, the formation of small particles. At low $\mathrm{pH}$ however, the system approaches the isoelectric point, which destabilizes the system. This results in higher rate of aggregation, forming larger particles.

\subsubsection{Capping Agent}

One of the traits of silver is its ability to oxidize spontaneously under standard conditions. Even nanoparticles are not immune to oxidation. Upon oxidation, the properties of the nanoparticles will diminish or change, which will affect their intended application. Hence, polymers and microemulsions have been used as capping agents to provide protection to the particles by coating them. In addition to protection, they are capable of altering the conditions of the product, including particle size, shape and rate of formation.

There are numerous polymers that have been studied for use as capping agents. Some commonly used polymers are polyvinylpyrrolidone (PVP), polyvinylalcohol (PVA) and polyethyleneglycol (PEG).

A study by Wang et al. was conducted to determine the mechanism and coordination of PVP to AgNP. ${ }^{17}$ The nanoparticles were synthesized by creating a solution of glucose, PVP and sodium hydroxide and adding a solution of silver nitrate to it drop wise. With the aid of the FTIR 
and UV-vis spectroscopy, it was determined that the PVP binds to both $\mathrm{Ag}^{+}$, which hindered the reaction, and $\mathrm{H}^{+}$, which helped in the progression of the reaction. Once $[\mathrm{Ag}(\mathrm{PVP})]^{+}$has formed, it would get reduced by glucose to form $[\mathrm{Ag}(\mathrm{PVP})]$. It was determined that the coordination of the PVP to the nanoparticles was dependent on the final size of the particles. For particles with diameters smaller than $50 \mathrm{~nm}$, the nitrogen of the pyrrolidyl would coordinate with the silver ion despite the steric hindrance. For particles between 500nm and $1000 \mathrm{~nm}$, both the oxygen and nitrogen coordinate to silver. Wang et al. have also demonstrated the control on particle size by manipulating the weight ratio of PVP or the silver acetate, with the increase in PVP weight ratio resulting in smaller particle production. In addition to protecting the particles from oxidation, PVP also prevents the possibility of a silver-silver particle bond from forming.

\subsubsection{Physical Synthesis}

The chemical synthesis methods provide simplicity through using readily available chemicals, in addition to having the convenience of using no expensive instrument for the creation of the particles. However, they do not provide completely pure silver particles since they requires the use of surfactants and stabilizers to perform the synthesis. ${ }^{4}$ Furthermore, some reducing agents may leave behind contaminants such as boron, nitrogen or carbon. Having pure AgNP are crucial for their applications.

The physical synthetic methods require little to no reducing agents. Hence, synthesized nanoparticles will be significantly purer than those obtained via the chemical method. Physical methods include photoreduction, laser ablation, electrolysis, ultrasonic irradiation and evaporation/condensation. 


\subsubsection{Gamma Rays}

There are many methods of synthesizing AgNP that fall under the photoreductive category, which include microwaves ${ }^{2}, \mathrm{UV}$-vis ${ }^{18}$ and gamma rays. ${ }^{19}$ The Gamma ray method is the most researched physical method since it offers excellent control on reaction rate. ${ }^{2}$ In addition, the radiolysis of aqueous solutions with gamma rays produces hydrated electrons, which are capable of reducing metals. This process negates the necessity of adding additional reducing agents. Irradiating water with gamma rays will produce $\mathrm{H}_{3} \mathrm{O}^{+}, \mathrm{H}, \mathrm{H}_{2}, \mathrm{OH}, \mathrm{H}_{2} \mathrm{O}_{2}$ and $\mathrm{e}_{\mathrm{aq}}{ }^{-}$ . The $\mathrm{e}_{\mathrm{aq}}{ }^{-}$will reduce any metal ion to its elemental form, which will aggregate to form nanoparticles.

A comparison between the chemical reduction method and gamma ray physical method for both silver and gold nanoparticles was conducted by Li et al. This comparison is demonstrated by the size of the particles and their concentration in solution. With the physical method, the concentration and of the nanoparticles produced can be increased by increasing the concentration of silver nitrate. The chemical method is also capable of producing particles with varied size and concentration by varying the concentration of $\mathrm{AgNO}_{3}$, however it was only successful with two specific concentrations. In addition, the chemical method produces particles with large size distribution. The physical method however, has a smaller size distribution, making it a more precise method when synthesizing a desired size. The dose of the gamma radiation also influences the final size of the particles; higher dose provides smaller particles, and lower dose give larger particles. ${ }^{20}$ 


\subsubsection{UV-Vis}

Photoreductive production of nanoparticles has also been achieved with UV and visible (UV-vis) radiation. However, most UV-vis methods require the use of a protective agent to control their size and stabilize them after production. ${ }^{21}$ Kshirsagar et al. have been successful at synthesizing nanoparticles with silver sulphate, L-tyrosine and potassium hydroxide under 266nm laser or 4W UV-A lamp irradiation. With the laser, an average of $8 \mathrm{~nm}$ sized particles were produced, while the lamp produced an average of 19nm AgNP. The size difference was attributed to the weaker intensity with UV-A lamp, causing slow reduction and a slow rate of nucleation. A high rate of reduction will result in the formation of smaller particles as it forms many nucleation sites with a small chance for growth. High intensity lasers may also cause larger particles to fragment into smaller ones. Thus, any large particles that were observed in the initial stages of Kshirsagar's reactions were no longer present when the system was analyzed. This is another example of how much control is present in the physical synthesis method.

While the majority of the UV-Vis nanoparticle synthesis mainly utilizes the UV spectrum, the visible does have some potential for excellent size and morphology control as shown by Stamplecoskie et al. ${ }^{22}$ A solution of $\mathrm{AgNO}_{3}$, citrate and compound I-2959 was exposed to UV-A, creating AgNP with an approximate size of 3nm. These nanoparticles are referred to as nanoseeds in their communication. The solutions were split into five individual cuvettes and exposed to $405 \mathrm{~nm}, 455 \mathrm{~nm}, 505 \mathrm{~nm}, 627 \mathrm{~nm}$ or $720 \mathrm{~nm}$ light emitting diodes (LEDs). This exposure results in the growth of the nanoseeds to spheres ( at $405 \mathrm{~nm}$ ), dodecahedra (455nm), hexagonal $(505 \mathrm{~nm})$, triangular $(627 \mathrm{~nm})$, and nanorods $(720 \mathrm{~nm})$. The particle sizes range between $5 \mathrm{~nm}$ and $25 \mathrm{~nm}$ depending on duration of exposure. 


\subsubsection{Laser Ablation}

Laser ablation is another effective method for synthesizing nanoparticles in solution. ${ }^{2}$ Synthesizing AgNP is accomplished by placing solid silver metal in solution, and exposing it to a high intensity later beam. The laser would fragment the solid silver into small colloids that would be suspended in water. Suspending the particles in water is usually aided by adding ions or

surfactants to the solution, to prevent the colloids and nanoparticles from self-aggregating. ${ }^{23,24}$ Such agents may also aid in providing control on the final size of the particles. Tsuji et al. have explored a two-step laser ablation method of synthesizing AgNP. A 99.99\% pure plate of silver was placed in pure water. In the first step, the plate was exposed to a Nd:YAG 1064nm laser beam for 10 minutes. This will result in creating colloidal silver suspended in the water. In the second step, the plate was removed from the solution, and then the solution was exposed to $\mathrm{Nd}$ :YAG $355 \mathrm{~nm}$ laser, which causes to colloids to fragment into smaller particles. The transmission electron microscope (TEM) was used to analyze the size of the particles before and after the $335 \mathrm{~nm}$ exposure. With only $1064 \mathrm{~nm}$, the particle size ranged between $10 \mathrm{~nm}$ and $100 \mathrm{~nm}$. After exposure to $355 \mathrm{~nm}$ laser, no particles larger than 50nm were observed. In addition, the formations of nanowires were observed, having a diameter between 10-100nm, and a length between $10 \mathrm{~nm}$ and $1 \mu \mathrm{m}$.

Mafune et al. made use of a surfactant in the production of nanoparticles through the laser ablation method. The effects of laser intensity and concentration of surfactant were studied in this experiment. Sodium dodecyl sulfate (SDS) was used as a surfactant in this experiment. A $99.99 \%$ pure piece of silver was placed in the bottom of a vial containing a solution of SDS. The solution was then exposed to 532nm laser. An increase in SDS concentration resulted in a decrease of average particle size. The laser intensity was kept constant. An increase in laser 
intensity however, with a constant SDS concentration, resulted in an increase in average particle size.

\subsubsection{Microwave irradiation}

The final irradiative method of nanoparticle synthesis is microwave irradiation. This method relies on the chemical method for synthesizing the nanoparticles, and improves it through the use of microwave radiation. ${ }^{25}$ The radiation is capable of heating up a solution at faster rates than conventional thermal methods. There are two methods of irradiating the samples with microwave radiation, fixed frequency microwave and variable frequency microwave (VFM). The VFM method is usually used since the penetration depth of the microwaves is reliant on the frequency and type of medium it is penetrating. By varying the frequency, the microwaves will penetrate the medium to different depths, allowing for a quicker and more uniform method for heating up the medium. In Jiang et al's study, the variable frequency microwave method is used to synthesize the nanoparticles from a $\mathrm{AgNO}_{3}$ / PVP solution. When the concentration of $\mathrm{AgNO}_{3}$ was increased, the size of the nanoparticles increases, in addition to the size distribution. The VFM method was compared to the conventional method of heating, in an oil bath, in terms of particle size and size distribution. It was determined that the VFM method of particle production has a narrower size distribution than the conventional method. The conventional method would require time for the solution to heat up all the way to its core. The VFM solved that problem, since it can penetrate the solution and heat it at multiple depths, providing a more uniform heat distribution and a shorter time. 


\subsubsection{Ultrasound}

Much like the microwave method, the sonochemical method relies on ultrasound to increase the systems temperature to aid in the production of nanoparticles. Due to the harsh events the ultrasonic waves create within a solution, this method could be performed without the use of a reducing agent. ${ }^{26}$ Upon exposure to ultrasound, acoustic cavitation occurs within the solution. These acoustic cavitations are simply bubbles in the liquid that will form, grow and then undergo an implosive collapse. This collapse results in formation of localized hotspots due to adiabatic compression or shockwave formation within the collapsed bubble. These hot spots cause the formation of extreme conditions, such as transient temperatures of $5000 \mathrm{~K}$, 1800atm of pressure, and a cooling rate of $10^{10} \mathrm{~K} / \mathrm{s}$. Such conditions are sufficient to reduce silver salts. Salkar et al. have successfully formed AgNP using the sonochemical method by creating a solution of silver nitrate and sonicating it for 1 hour under argon-hydrogen atmosphere. TEM images have revealed that the particles were approximately $20 \mathrm{~nm}$ in size and amorphous in shape.

The mechanism of the reaction depends on the formation of radicals that aid in the reduction of $\mathrm{Ag}^{+}$to $\mathrm{Ag}^{0}$. These radicals are formed by the sonochemical reaction, splitting the water molecule to $\mathrm{H}^{\bullet}$ and $\mathrm{OH}^{\bullet}$. The $\mathrm{H}^{\bullet}$ radical will reduce $\mathrm{Ag}^{+}$to $\mathrm{Ag}^{0}$. This is then followed up by the aggregation of silver to form the particles.

This method does have its drawbacks however. The shape produced was characterized as amorphous, and it does not appear that there is a way to control the shape of the particle without the aid of a capping agent. Also, it is necessary to contain the reaction in an oxygen free 
environment to avoid the re-oxidation of the produced silver. In addition, it is possible for $2 \mathrm{OH}^{\bullet}$ to form $\mathrm{H}_{2} \mathrm{O}_{2}$, which could oxidize the silver to $\mathrm{Ag}_{2} \mathrm{O}$.

\subsubsection{Synthesis on a solid surface}

All the methods discussed above yield nanoparticles in solution. Some applications, such as SERS, require the particles to be adhered onto a solid surface. There are two ways to achieve this, either synthesize the particles directly onto a solid surface, or synthesize them in solution, followed by depositing them on the desired surface.

\subsubsection{Electrostatic interaction}

Li et al. have developed two novel method of adhering AgNP onto a quartz substrate through means of electrostatic interaction. ${ }^{27}$ Both methods begin by synthesizing AgI nanoparticles by reacting $\mathrm{AgNO}_{3}$ with mercaptoacetic acid and sodium iodide (NaI). This is followed by layering quartz slides with $\mathrm{OH}^{-}$and then a layer of poly(diallydimethylammonium

chloride) (PDDA). Method one begins by dipping the layered plate into the AgI nanoparticles to create a layer of nanoparticles on the plate. This is followed by immersing the plate in a solution of $\mathrm{NaBH}_{4}$ to reduce the $\mathrm{AgI}$ nanoparticles to $\mathrm{Ag}^{0}$. Method two involves reduction of the $\mathrm{AgI}$ with $\mathrm{NaBH}_{4}$ while still in solution, and then dips the slide into that solution to create a layer of AgNP. Multiple layers can be applied to the same plate by alternating in dipping between PDDA and Ag solutions. Method one was determined to have a superior SERS signal when compared to method two. It is believed that the mercaptoacetic acid in method two blocks the active sites on the surface, weakening the SERS signal. This shows that the purity of the nanoparticles impacts their applications with SERS; the cleaner the particles, and stronger the SERS signal. 
A similar method was developed by Jia et al, in which silver nanoseeds were deposited onto the slides, and allowed to grow. The nanoseeds were synthesized by adding a solution of sodium citrate to a silver nitrate solution with continuous stirring. Then sodium borohydride was added. The slides were treated with PDDA solution, dipped into the silver nanoseed solution for 10min, and then dipped into a fresh solution of silver nitrate and sodium citrate. The slide was then irradiated with a sodium lamp.

\subsection{Becquerel Plate}

As discussed in the previous section, many methods exist for synthesizing silver nanoparticles. Most of these methods accomplish the synthesis in solution form, while additional methods are used to adhere the particles on a solid surface. Any applications that require the particles to be adhere on the surface, as is the case with SERS, will have include those additional methods.

We propose a method of synthesizing AgNP directly onto a silver plate through the use of the Becquerel Method. In this section, we will discuss the history of the Becquerel plate, and the experiments that will be conducted to study the parameters that impact particle size, shape and morphology.

\subsubsection{History}

The history of the Becquerel plate can be traced back to the year 1840, shortly after the invention and popular adoption of the daguerreotype process. ${ }^{28}$ The daguerreotype was the first method of photography that was available in the market for use by the public. Introduced in 1839 , the daguerreotype was invented by Louis Daguerre. Its popularity arises from its ability to create accurate portraits or images, without the need of an artist. And thus, it became quite 
popular all across Europe and North America. The era of the daguerreotype lasted until 1860 when it was superseded by the technically simpler processes that also permitted reproduction.

The concept of creating a daguerreotype is quite simple, but the technical methodology is difficult to perfect. A silver plate, or copper plate electroplated with silver, is cleaned and polished to a mirror shine and washed with alcohol or dilute nitric acid to dissolve away any grease or grime left behind during the polishing process. The plate is then placed into an iodine chamber, to form a thin surface layer of silver iodide. The sensitized plate is inserted into a camera, and exposed. After exposing the plate, the photographer would maintain the plate in darkness to preserve the latent image and then develop it by exposing it to mercury vapour. The developed plate would be washed with distilled water and $3 \%$ sodium thiosulfate, to dissolve away the remaining silver iodide. The result would be a positive image comprised of nanoparticles (white or "highlight" areas) of silver-mercury amalgam. The particles were often further fixed by treatment with gold(III) solution.

Shortly after Daguerre described his technique, Edmond Becquerel discovered a new method for developing the images without the need of mercury. This was accomplished by exposing the plate with the latent image to high-intensity red or yellow light for 10 minutes. In comparison to the daguerreotype, the Becquerel image has less contrast, and a slight blue tint. Recent analysis of Becquerel plates with scanning electron microscopy showed that the plates are covered with spherical nanoparticles. X-ray analysis of the particles revealed that the nanoparticles are silver(0).

Despite the technological applications of silver nanoparticles, little attention has been paid to the Becquerel technique for creating them. Most research on Becquerel plates has been 
carried out by investigators in the visual arts, and their focus has been on improving or maintaining the images. The Becquerel technique holds some promise technologically as it is essentially a "dry" method of producing patterned nanoparticles directly on a surface.

\subsubsection{Studying the Becquerel method for nanoparticle synthesis}

The Becquerel process involves a number of steps with several parameters that can influence the process outcomes; for example, the thickness of the silver iodide layer, age of the silver iodide layer, wavelength and intensity of the excitation, delay between excitation and development, and wavelength and intensity of development light.

Irving Pobboravsky studied the iodization of daguerreotypes. ${ }^{29}$ The purposes of this study were primarily to understand and improve the daguerreotype method. It was observed by daguerreotype enthusiasts that the quality of the images produced depends on the silver iodide layer. The optimum thickness for exposure is identified by the colour of the plate. When the plate colour is yellow with a hint of magenta, the plate would provide a good quality image. Under exposing a plate with iodine provides a colour closer to the silver colour, which would result in a faint image or no image at all. Over exposing a plate, which usually yields a colour that would be heavy magenta, blue, or black, would also provide a low quality image. Pobboravsky has used numerous methods, including X-ray fluorescence and spectral absorbance, to determine that the thickness of silver iodide that gives a yellow-magenta colour to be approximately $30 \mathrm{~nm} .{ }^{29}$ This effective thickness also applies to the Becquerel method. For the purposes of this study, the effects of silver iodide thickness on the particle formation will not be explored.

The age of the silver iodide was explored in this paper. Mike Robinson reports that the quality of the image is affected by the age of silver iodide. When comparing the image of a plate 
that was freshly sensitized with silver iodide to a plate that had 30 minutes old silver iodide, it was observed that the image with 30 minute old silver iodide was clearer, with better contrast. This aging effect may not be limited to the daguerreotype method only, which may become a crucial variable in the formation of the particles. Analysis of this parameter will be done exclusively with the SEM.

The excitation wavelength or band is the one parameter that influences the creation of nanoparticles. Studying its influence on their size, shape and morphology is important in understanding the mechanism of formation of the particles. ${ }^{22}$ The central wavelength and intensity of the excitation band was controlled in this work through the use of eight highintensity LEDs. Each LED had a narrow wavelength distribution (approx. $30 \mathrm{~nm}$ ) and a variable intensity controlled by a potentiometer. A calibrated thermopile was used to normalize the intensities of the LEDs.

After excitation the plates are usually exposed to red light to aid in the growth of the particles( development step). It is possible that the time period between excitation and growth steps will have an effect on the particles produced. With daguerreotypes, it is typically best to develop as soon as possible, to prevent the image from degrading; waiting too long results in complete image loss. Since our study is on particles and not image quality, this could be a useful parameter to control

Much like the excitation step, the red light development may also play a role in the varying the type of particle that is produced. Both intensity and wavelength may play a role in the particle development. Hence, like with excitation step, the wavelength and intensity will need 
to be controlled to study these effects. Once again, the same LED array will be used to help in making this determination.

The final step of the Becquerel method is the fixing method, exposing the plate to $3 \%$ sodium thiosulfate. This step is primarily meant to remove any excess unreacted silver iodide left behind to prevent unintended conversion of this photoreactive material to elemental silver. 


\section{Methods}

\subsection{Specialized equipment}

A certain level of control is required in order to get the desired results. Some equipment was designed and fabricated in-house to achieve that level of control. The creation of both the iodine chamber and LED array will be discussed in this section

\subsubsection{Iodine Chamber}

The iodine chamber is made up of three pieces, a jacketed beaker, a beaker adaptor, and a plate holder. A $500 \mathrm{~mL}$ jacketed beaker was used to serve as the chamber, to store the iodine crystals. The beaker was attached to a VWR circulating bath. The bath was set to $20^{\circ} \mathrm{C}$ for all iodization techniques. Both the beaker adaptor and plate holder were created through the use of a 3-D printer. They were designed using Solidworks version 5, and printed using Dimensions 1200es.

The beaker adaptor was designed to fit tightly onto the jacketed beaker (Figure 2.1), a square top with a length of 3.75 inches (Figure 2.3). A 3.25 inch long slit with a $0.025 "$ thickness slit running through

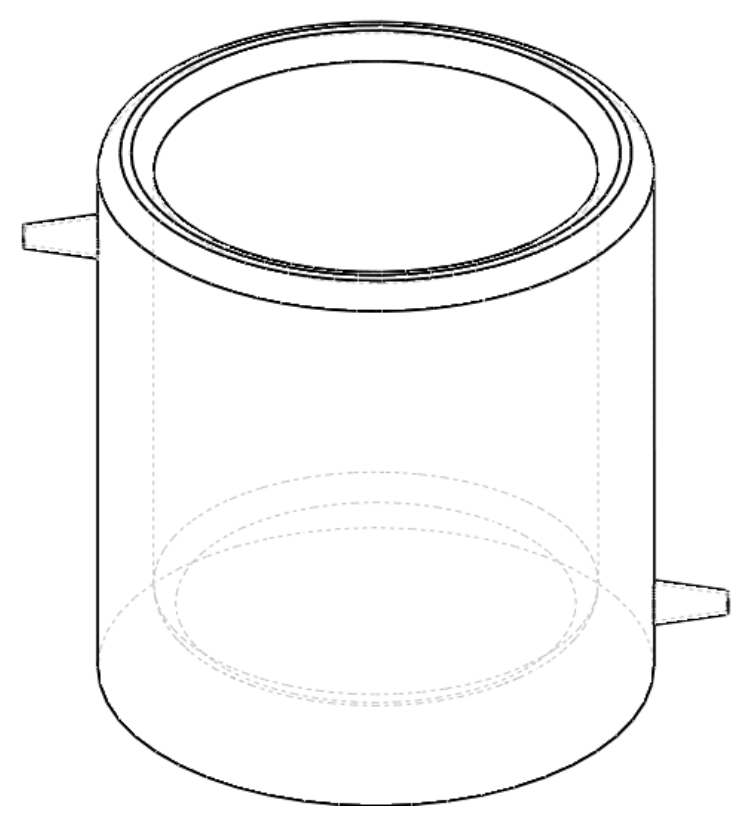

Figure 2.1: Jacketed beaker for containing $I_{2}$ and controlling the temperature of its vapor. 
it was also designed. A 316 grade

stainless steel sheet was purchased from

amazon.com. It was cut to a width of 3.25

inches to fit through the slit. This served

as the shutter to the chamber, when closed

it allows the buildup of iodine vapour

equilibrated to the set temperature within

the chamber. When open, it will release

the vapour to expose the plate to iodine.

The plate holder was designed

with the same square shape as the beaker adaptor, square with 3.75 inch in length, and to fit directly on top of the beaker adaptor (Figure 2.2). In addition, a small groove with the dimensions of 2.5 inches by 2 inches was designed to place the plate, and a hole through it that has a 0.25 think frame to hold the plate.

Furthermore, an identical slit to the beaker adaptor was also created below the plate's level, with an identical stainless steel sheet running through it. This shutter serves primarily to protect the plate from
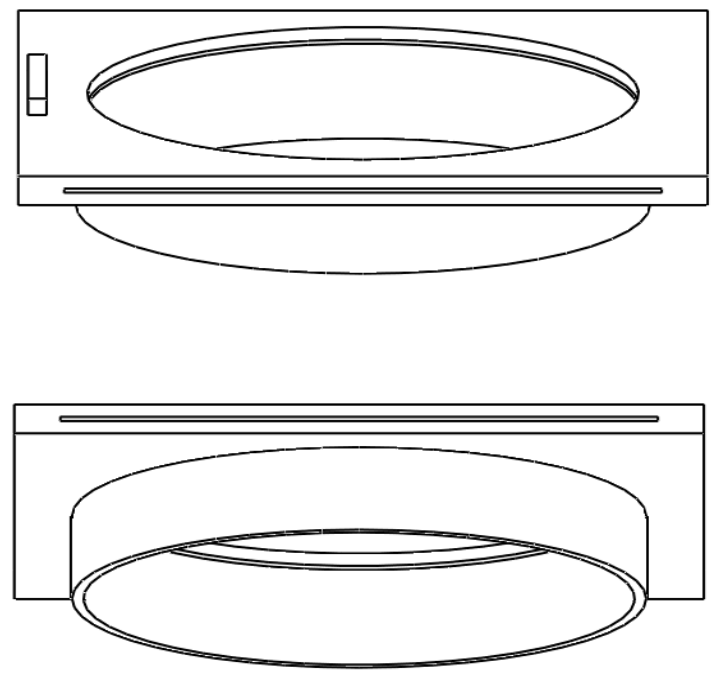

Figure 2.3: The adaptor that connects the plate holder and the iodine chamber together. Top image shows the top view, and bottom image shows the bottom view
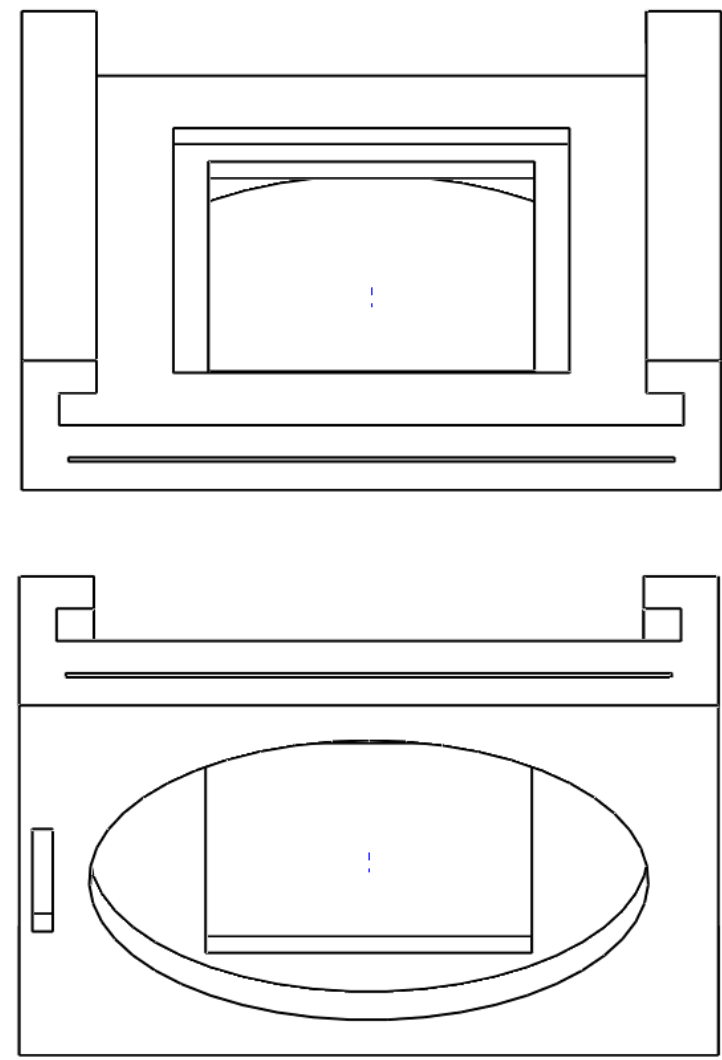

Figure 2.2: The plate holder. The top image is the top view, the bottom is a bottom view. 
light after it has been photosensitized with iodine vapour. This also allows the user to move the plate from place to place without the concern of light exposure.

\subsubsection{LED Array}

An LED array was designed to allow for controlled light exposure onto the silver plates. A 3 inch by 1 inch aluminum L angle, with a length of 3 feet was purchased from Metals Supermarket. The aluminum $\mathrm{L}$ angle was cut to two pieces, 1 foot long, and 2 feet long. The one foot long section was used to hold the detector, a calibrated thermopile purchased form Dexter industries. The two feet section was used to hold the LEDs in place. The two pieces were designed to be used on an optical bench with imperial measurements. Each of the screw holes have a 1 inch separation.

For the first section, a two inch long circular straight slot was drilled at the center of the 3 inch side of the aluminum L angle (Figure 2.4). The slot has the thickness of the thermopile's

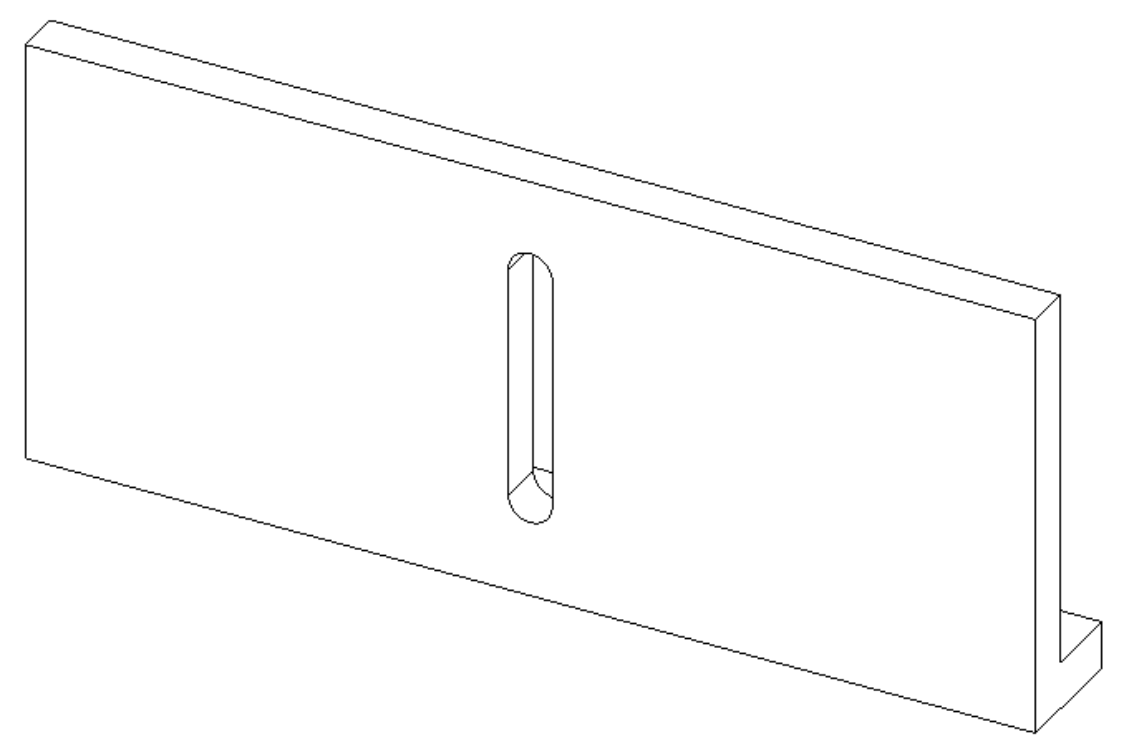

Figure 2.4: A front view of the Thermopile holder. The centre vertical slit is where the thermopile is mounted, allowing for vertical translation. 
body, and serves to hold the thermopile in place and allow for vertical translation. The aluminum $\mathrm{L}$ angle was spray painted with a matte black finish. On the 1 inch side of the aluminum $\mathrm{L}$ angle, two circular straight slots were drilled with the length of 5.5 inches, separated by 0.5 inches from one another. The width of the slot is large enough to allow $1 / 4$ inch screws to fasten the aluminum $\mathrm{L}$ angle to the optical bench. The slots will also allow for side to side translation of the thermopile.

The second section of the aluminum L angle was used to hold the LEDs in place. A total of eight 1 inch diameter holes were drilled at the front face of the aluminum L angle, separated by 3 inches. The 1 inch side had a total of 9 holes drilled into it, with $1 / 4$ inch diameter to fasten it to the optical bench. The holes were separated by 3 inches. Eight narrow band LEDs were purchased from www.luxeonstar.com, with wavelengths of 447nm, 470nm, 505nm, 530nm,

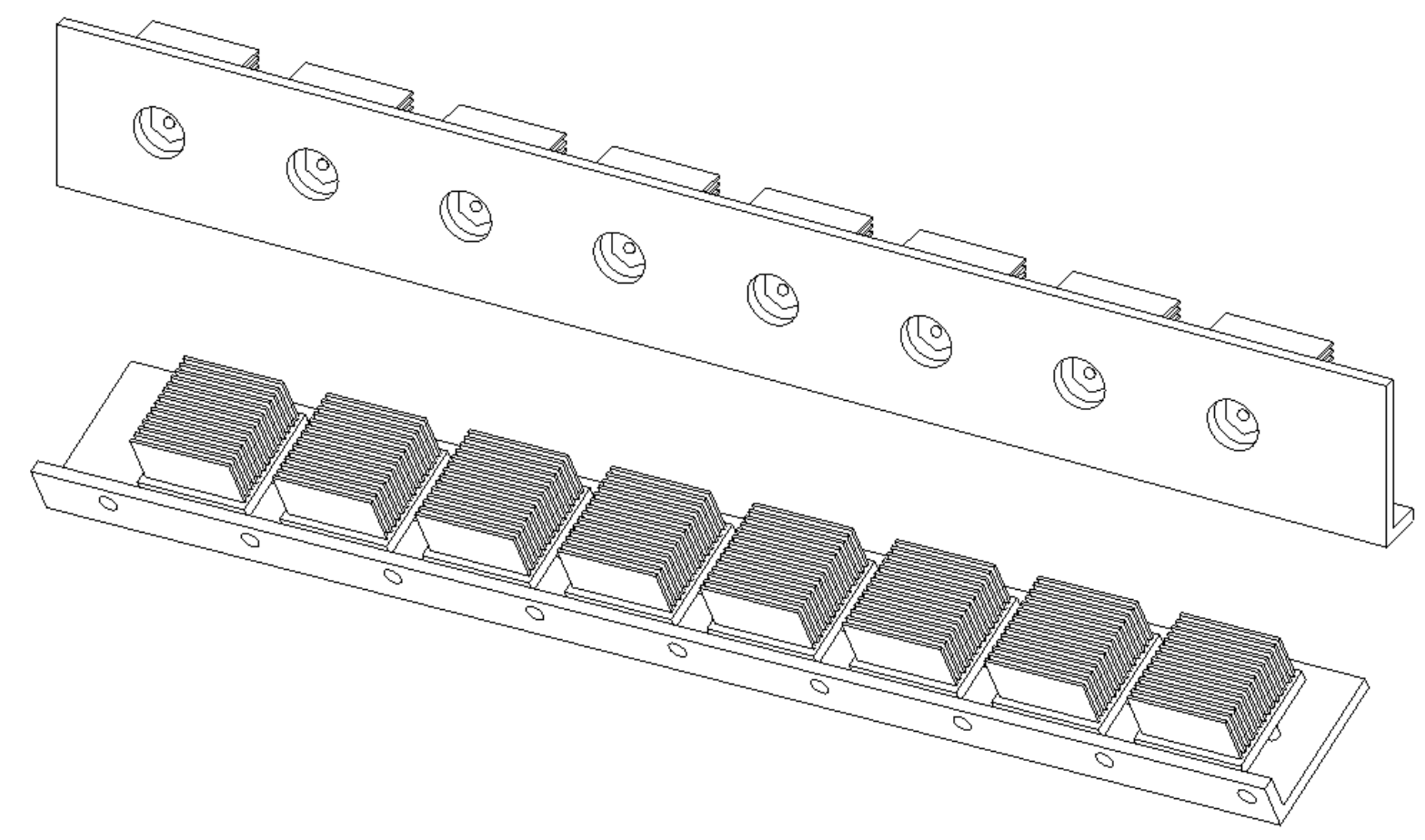

Figure 2.5: LED array, with wavelengths of 447nm, 470nm, 505nm, 530nm, 591nm, 617nm, 627nm, and 655nm. The top is the front view, and the bottom is the rear view. 
$590 \mathrm{~nm}, 617 \mathrm{~nm}, 627 \mathrm{~nm}$, and $655 \mathrm{~nm}$. Each LED was attached to the centre of aluminum heat sinks with heat conducting double sided tape provided by Luxeon star. The wires were then soldered onto each lead of the LEDs and to a potentiometer. Two $1 / 4$ inch stainless steel nuts were glued onto each heat sink using metal adhesive. Once dried, each heat sink was glued onto the aluminum $\mathrm{L}$ angle with the LED facing dead center to 1 inch drilled holes. The LEDs were glued in order from lowest to highest in wavelength. Figure 2.5 shows an image of the final product.

\subsection{Plate Preparation}

\subsubsection{Polishing}

NuShine S grade polish was used for polishing all silver plates. A small portion of NuShine (about $0.5 \mathrm{~mL}$ ) is placed onto the plate. The NuShine is then diluted down with hexane. The polish is then rubbed against the plate with moderate pressure with cotton velvet fabric. Once polished to a mirror finish, the plate was washed with hexane and rubbed down with hexane soaked velvet fabric until no polish residue remains. After washing with hexane, the washing process was repeated again with detergent, water, then with ethanol.

\subsubsection{Iodization of the silver plate}

The temperature control of the iodine chamber was set to $20^{\circ} \mathrm{C}$, and given 30 minutes to equilibrate. A highly polished plate is placed face down onto velvet cloth. A Ziploc bag filled with water at $20^{\circ} \mathrm{C}$ was placed on top of the plate. After 10 minutes, the plate was placed on top of the iodine chamber and exposed to iodine vapour for 4 minutes. Once 4 minutes had passed, the iodine chamber and the plate holder were sealed, isolating the surface from the iodine vapour and protecting it from light during transport from chamber to instruments. 


\subsection{Experiments}

\subsubsection{Excitation study}

\subsubsection{Red light Developed}

\subsection{Visible Study}

Each plate was polished and iodized as described under "plate preparation". LEDs emitting at $447 \mathrm{~nm}, 470 \mathrm{~nm}, 505 \mathrm{~nm}, 530 \mathrm{~nm}, 590 \mathrm{~nm}$ and $617 \mathrm{~nm}$ were used to form nucleation sites. Using the thermopile, all the intensities of the LEDs were set to have equal photon fluxes (photon counts per unit area per unit time). The plate was divided into 6 regions, one for each of the LEDs. The thermopile was then removed from its holder, while the plate was set up against the slit, to ensure consistent exposure. The 447-nm LED was turned on for 5 seconds directed at a cardboard shutter, and then directed onto the plate for 10 seconds (initiation), followed by a 1 minute delay, and then a 10 minute exposure to the 655-nm LED (development). The plate was shifted to the next region, and all steps repeated, except that the 470-nm LED was used for initiation. This process was repeated with all the LEDs. Once all the regions were developed, the plate was removed from the slit, and exposed to $3 \%$ sodium thiosulfate. The plate was then washed with Milli-Q water, air dried and stored into a vacuum chamber. The plate was cut into 6 portions using a band saw, each portion a sample for each of the LED exposures. The samples were all analyzed by SEM.

\subsection{UV light with mercury arc lamp}

Each plate was polished and iodized as described under "plate preparation". An Orion made 350W mercury arc lamp, model 66033, was used as the source. The lamp had an adjustable 
condensing lens, and a water filled IR filter. The most intense wavelengths emitted by mercury arc lamp were used, including $406 \mathrm{~nm}, 366 \mathrm{~nm}, 334 \mathrm{~nm}, 314 \mathrm{~nm}, 302 \mathrm{~nm}$, and $254 \mathrm{~nm}$. The plate was split into 6 regions for each of the exposure wavelengths. The lamp was positioned to shine its light directly into the slit of a monochrometer to control and isolate the light to strike the plate. The lamp was turned on and allowed to warm up for 15 minutes. The thermopile was used to measure the intensity of the light for each of the chosen wavelengths. The thermopile was moved and the iodized plate was put in its place. The $406 \mathrm{~nm}$ light was allowed to strike the plate for 10 seconds, followed by exposure to $655 \mathrm{~nm}$ light from the LED array for 10 minutes. This process was repeated for the rest of the chosen wavelengths, but each wavelength was used to expose a different region of the plate. Once all the regions have been exposed, the plate was submerged in a pool of $3 \%$ sodium thiosulfate for a few minutes, washed with $18.2 \mathrm{M} \Omega$ water, and air dried. The plate was then cut into 6 pieces, 1 piece for each region, and analyzed using the SEM.

\subsubsection{Undeveloped with red light}

Each plate was polished and iodized. All LEDs were used for this experiment. Using the thermopile, all the intensities of the LEDs were set to equal photon counts per second per square centimetre. The plate was divided into eight regions for each of the samples. Each region was exposed to one of the LEDs for a total of 4 minutes. The experiments began with $447 \mathrm{~nm}$, and ended with $655 \mathrm{~nm}$ LED. There was a 1 minute rest between the exposure of each sample to adjust the plate, and LED position. After all samples have been created, the plate was submerged in 3\% sodium thiosulfate, washed with Milli-Q water, and air dried. All samples were analyzed using the SEM. 


\subsubsection{Development Study}

Each plate was polished and iodized as per the steps under "plate preparation" subheading. LEDs $655 \mathrm{~nm}, 627 \mathrm{~nm}, 617 \mathrm{~nm}, 590 \mathrm{~nm}, 530 \mathrm{~nm}$ and $505 \mathrm{~nm}$ were investigated for use in nanoparticle growth. Each LED's intensity was adjusted to have an equal photon count between all of them, with the aid of the thermopile. The excitation step was performed using the 447nm LED light. It was set at full intensity. The thermopile was removed from its holder, and the plate was set up against its slit. A total of 6 regions were marked on the back of the plate to indicate were each sample was to be created. The 447nm LED was turned on with a piece of cardboard in front of it acting as a shutter. The shutter was lifted after 3 seconds to allow exposure of the plate. After 3 seconds of exposure, the shutter covered the LED once again and the LED was turned off. After a 60-second delay, the same region of the plate was exposed to the $655 \mathrm{~nm}$ LED for 10 minutes. This concluded the preparation for the first sample. The plate was then shifted to the next region to prepare the next sample. The same steps were repeated, except that a different "growth" LED (627-nm, 617-nm, 590-nm, 530-nm and 505-nm) was used. Once the preparation of all 6 samples was complete, the plate was submerged into a petri dish filled with 3\% sodium thiosulfate and washed with Milli-Q water. The plate was air dried and stored in a vacuum chamber for later analysis by SEM.

\subsubsection{Iodine Maturation Study}

\subsubsection{Iodine Investigation}

A plate was polished as per the "polishing" method under "plate preparation"

subheading. The plate was then scored with a utility knife to divide it into 4 quadrants. This was followed by iodization of the plate as mentioned under "plate preparation". With a paper shear, 
the plate was cut into 4 quadrants, using the scored markings as a guide. This was all done in the dark room. Three pieces were placed into a container, and stored in a dark cupboard. The fourth piece was immediately washed with $5 \mathrm{~mL}$ of $3 \%$ thiosulfate, and the washings collected in a clean petri dish. A Pasteur pipette was used to recycle the $5 \mathrm{~mL}$ thiosulfate against the plate multiple times to ensure all silver iodide was completely removed. The $5 \mathrm{~mL}$ thiosulfate was then transferred to a $10 \mathrm{~mL}$ volumetric flash. Fresh thiosulfate was used to wash the plate one last time. This was also used to rinse the Petri dish before transferring to a $10 \mathrm{~mL}$ volumetric flask, and then topping to the mark with fresh thiosulfate. The flask was stored in a dark cupboard. The procedure was then repeated with the remaining 3 slices once they had matured for 1,3 and 5 days. This entire procedure was repeated with an additional two plates, to give a total of 12 samples for analysis. All samples were then analyzed using the ICP.

\subsubsection{Particle investigation}

Three plates were polished and iodized. For each plate, four samples (one in the centre of each quadrant) were prepared with a set time period separating the preparation of each sample. The first sample of each plate was prepared shortly after iodization. This serves as the control. The sample was prepared by exposing it to 447-nm LED for 10 seconds, followed by a $1 \mathrm{~min}$ rest, then 655-nm LED for 10 minutes to begin the growth process. The following samples were prepared a set time period after the growth step was complete. The time period was 1 minute for plate one, 1 hour for plate two, and 1 day for plate three. Once all 4 samples have been created, the plate was submerged in 3\% sodium thiosulfate, washed with Milli-Q water, air dried, and stored in a vacuum chamber. The plates were analyzed using SEM. 


\subsection{Instrumental Analysis}

\subsubsection{ICP-AES Analysis}

For ICP analysis, standards were created using aqueous silver acetate and potassium iodide (KI). $100 \mathrm{ppm}$ of silver acetate was created by dissolving 0.1 grams into $1 \mathrm{~L}$ volumetric flask and made to the mark with Milli-Q water (18.2 M $\Omega)$. A 1L flask of KI was creating with equimolar concentration to the silver acetate. 1ppm of silver standard solution was created by adding $1 \mathrm{~mL}$ of the stock silver acetate to a $100 \mathrm{~mL}$ volumetric flask, followed by adding $50 \mathrm{~mL}$ of $6 \%$ sodium thiosfulate, and then $1 \mathrm{~mL}$ of KI stock solution. Stock solutions of $3 \mathrm{ppm}, 5 \mathrm{ppm}$, $7 \mathrm{ppm}, 9 \mathrm{ppm}$ and $10 \mathrm{ppm}$ were created using the same method. $3 \%$ sodium thiosulfate was used as the blank solution.

The spectroflame compact E ICP AES was used for the analysis, with use of the smart analyzer 2.25 software. The $328.064 \mathrm{~nm}$ line was used for analyzing silver present.

\subsubsection{Scanning Electron Microscope (SEM) Analysis}

The SEM was used to analyze the size, morphology and how densely packed the particles were. Each sample was cut into approximately $1 \mathrm{~cm}^{2}$ in size using a band saw, to fit on the microscope's die. Each slide was attached to the die using double sided conductive tape. The magnification varied between $5000 \times$ to $50000 \times$ from sample to sample, depending on the size and how packed the particles were. An acceleration voltage of $20 \mathrm{kV}$ was used. All images were saved in .tif format. All the images were analyzed using the program ImageJ. The analysis on the images includes particle counts per unit area, particle size, and percent particles per unit area. 


\subsubsection{Particle analysis}

Each .TIF file was opened in ImageJ for analysis. Using the square selection tool, the scale bar was selected from side to side. This was done while zoomed in heavily on the scale bar to ensure that all the pixels have been selected. In the "image $\rightarrow$ show info..." tool, the number of pixels is listed for the selected item. In the "Analyze $\rightarrow$ set scale" tool, the scale was set with respect to the scale bar in pixel length. This will insure that any outputted data that have a distance measurements to it will be displayed in the desired units (such as area or particle size).

Next, the image was inverted, causing the particles to appear dark. The "process $\rightarrow$ background subtraction" tool was used to help bring out the particles that are faint that could be mistaken for background. Next, the thresholds of the images contrast were set at a level in which all visible particles remain visible, and eliminated any part of the image that could be mistaken for a particle. This will change the image from an 8-bit image to a binary. The image is then treated with a median filter, to remove any small specks that could be mistaken for particles, in addition to cleaning up the edges of the particles to appear less grainy. This helps in outputting a more accurate value for particle size or number of particles. The image is then treated with the "process $\rightarrow$ binary $\rightarrow$ watershed" tool. This tool helps separate any overlap between two particles that are next to each other, but appear as 1 particle in the image. The separation is accomplished by drawing a 1 pixel wide separation between the particles. While this tool does help to distinguish between two separate particles, it isn't perfect, and may miss some particles that do appear to be a combination of two particles. The image is then analyzed for particles using "analyze $\rightarrow$ analyze particles". In that menu, particle size was filtered for 150-infinity. The summary of the image was saved, which included details of the number of particles, total area 
and $\%$ area that covered the surface with particles. All the experimental data were tallied together into one file, and analyzed in Microsoft Excel. 


\section{Results and} Discussion

\subsection{Excitation Study (Development)}

To compare the difference between each LED's effects on the final product of the particles, the SEM images will be analyzed based on number of particles per unit area,

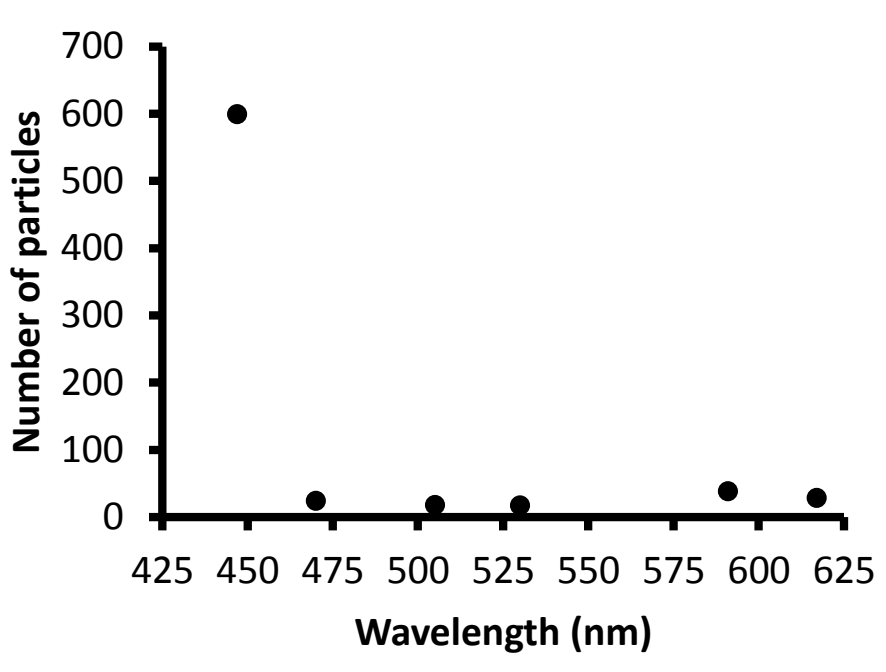

Figure 3.1: A graph of the number of particles produced by each LED during the excitation study.

and average size of the particles. Since the number of photons emitted from all the LEDs have been set to be identical to one, it is possible to make accurate conclusions based on the produced results without the need to normalize.

\subsubsection{Visible Study}

With regards to number of particles, the 447-nm LED produced the highest number of particles, as seen with the graph in Figure 3.1. The other LEDs produced fewer particles at 5\% or less of the total 447-nm LED. With 617-nm LED, the distance separating the particles is approximately $0.5 \mu \mathrm{m}$, while $447-\mathrm{nm}$ particle separation is on the order of a few nanometers. The rest of the LEDs yield a particle separation that is on the order of a few micrometers instead.

The use of different wavelengths in the excitation step to produce the particles has provided some varying results on the characteristics of the particles. This level of control is beneficial as it may widen the application and uses of such particles. For example, with regards to using SERS, a more densely packed plate will provide a better signal than a less densely 
packed plate. The more particles there are, and closer the particles are to one another, the greater the number of LSPR. The more LSPR there are, the greater the SERS signal, the greater the method's sensitivity. Based on this experiment, it is advised to use 447-nm or lower, if a higher particle density is desired.

The observation that 447-nm was the only wavelength that was successful at producing densely packed nanoparticles, may provide insight onto the mechanism of how the particles are produced. There were two mechanisms that were proposed for the creation of the particles. The first involves the silver substrate in the mechanism, where the light is absorbed by the substrate rather than the silver iodide. An electron would be excited to a higher state, and accepted by a silver iodide that is situated directly at the substrate. The second mechanism is mediated directly through the silver iodide layer: The incident light is absorbed by silver iodide, causing an electron transfer from iodide to silver, forming the $\operatorname{Ag}(0)$ nucleation sites. This is then followed by growth. The spectrum of silver iodide, transmits all light from approximately $450-\mathrm{nm}$ and higher. ${ }^{30}$ Below 450-nm, the absorbance rises sharply. Since nanoparticles are formed more effectively at $447-\mathrm{nm}$ and below, the latter mechanism is more likely.

The average particle size was unaffected by the initiation wavelength. In the first trial, the average particle sizes appear to increase as the wavelength increased, with 655-nm LED producing the largest particles, while 447-nm LED had the smallest size particles. LED 530-nm does not seem to follow this correlation. On other hand, the second trial had no present pattern correlated between the wavelength particle size. However, the 617-nm LED still did produce the largest sized particles. 
In the first trial, the particle size did seem to increase linearly with the wavelength. Both trials did reveal however, that $617 \mathrm{~nm}$ produced the largest of the particles. It isn't entirely clear as to why this occurs. One thought that may explain this is related to the effect of light energy on the particles. According to Nair et al, high energy light is usually used for formation of the nucleation sites, and low energy light is used for growth. While the $447 \mathrm{~nm}$ light may be capable of producing more nucleation sites, the rest of the LEDs are able to produce some nucleation sites. In addition to creating nucleation sites, $617 \mathrm{~nm}$ is also capable of inducing growth of the nucleation sites simultaneously. This results in more growth for those particles.

The development with 655-nm light, which results in the growth of the particles, is about 10 minutes long. While the exposure of $617 \mathrm{~nm}$ light is 10 seconds long, it is unlikely that these 10 seconds would cause sufficient growth for a difference in particle size to be noticed. Other undetermined factors are likely at play here.

In the case of 447-nm, having such small sized particles is understandable considering the number of nucleation sites produced. The final size of the particle will be dependent on the environment around it. The nucleation sites rely on the silver iodide surrounding them as the source of the silver when undergoing growth. When the nucleation sites are farther apart, the environment around it will be used strictly for that one nucleation site to grow. When the sites are closer to one another, then the surrounding environment is shared between multiple sites, reducing the amount of silver that is available for growth at each site. This will directly affect the final size of the particle. Since the results of 447-nm LED excitation yielded particles closely packed to one another, it is expected that their particle size would be smaller than the particles produced by the rest of the LEDs. This could also be another level of control that the user may have when producing a desired particle size. 


\subsubsection{UV study}

The visible study involved excitation wavelengths of 447-nm and higher. The UV study utilized wavelengths between 406-nm and 254-nm. With the LEDs, it was possible to control the intensity of the excited radiation, and have them match one another in photon counts. That way, the number of photons that strike the plate for a particular study would be nearly identical to one another, and a direct comparison between the results can be obtained. Any effects on size or density of the particles would be due to the difference in wavelength only. Hence, any conclusions made using this method would be sound.

UV-LEDs do exist in the market. The majority of them were available at approximately 385-nm to 400-nm. There weren't a wide variety of narrow band LEDs below 385-nm available in the market, and the ones that exist are quite expensive. A mercury arc lamp was available in the lab. With the use of the monochrometer, separating the UV light to selective narrow bands is possible. Selective control on the intensity of each wavelength however, was not possible with that setup. Each wavelength will have varying flux, and we will not have the same kind of control that we did with the visible study. Hence, comparison in particle counts in this case may not be controlled well, and no valid conclusions may arise from such data. The data will then have to be normalized with respect to the number of photons. The data will be analyzed as particle produced per photon, or photons per particle.

When analyzing the particles produced per photon, it can be observed in Figure 3.2 that this value begins at a high value between wavelengths $406-\mathrm{nm}$ and $334-\mathrm{nm}$. There is very little change between all 3 points. Below 366-nm, the particle per photon value begins to decrease, 


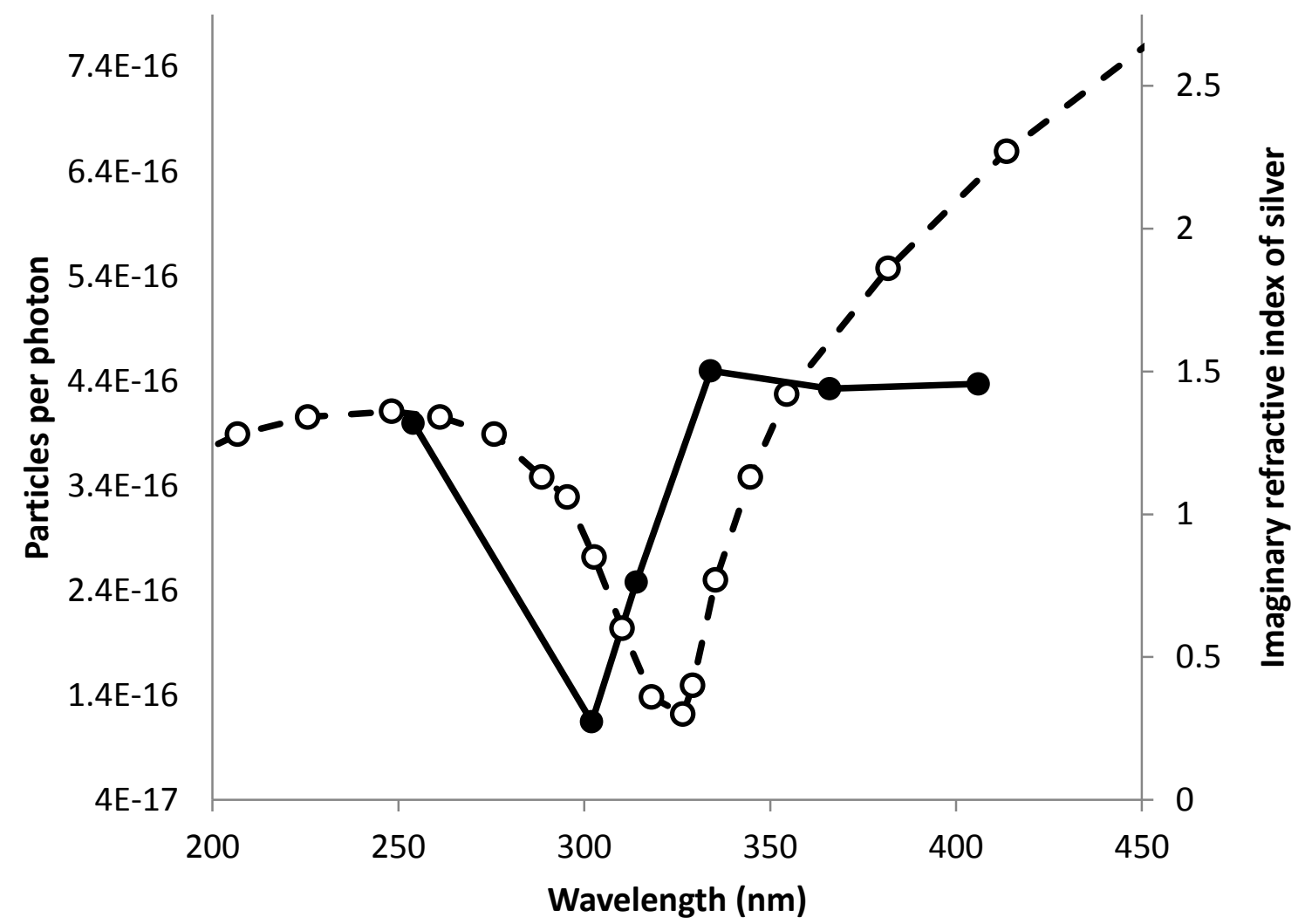

Figure 3.2: Particles per photon (solid dots, solid lines) for wavelengths 406-nm through to 254-nm compared to imaginary refractive index of silver metal (hollow dots, dashed lines)

with $302 \mathrm{~nm}$ having the lowest value between all the data. The value begins to rise once again at approximately $254 \mathrm{~nm}$.

This curve pattern that is observed doesn't seem to follow the silver iodide absorption, as it did with the visible side. Kurdyumova et al. report that silver iodide should continue to increase in absorption as the wavelength decreases. ${ }^{31}$ Yet particles produced per photon declines after 334-nm, then proceeds to rise after 302-nm. This may suggest that a second mechanism other than simple photon absorption is involved in the particle production.

When comparing the particles per photon curve to the curve of imaginary refractive index of silver metal, a slight resemblance is observed (imaginary refractive index is also proportional 
to the absorption of the material). The lowest point of the curve is at 326-nm, which begins to climb again at deep UV to a maximum of $250-\mathrm{nm}$. The particles per photon curve however, is shifted by approximately $20-\mathrm{nm}$. With such a shift, any conclusions that arise from a relationship between the imaginary refractive index and particles per photon reading may be unfounded, but other factors may be responsible for this shift.

One factor could be the silver iodide layer. In order for an absorption to occur at the silver layer, the incident ray will have to first pass through a thin layer of silver iodide. Kurdyumova has determined that silver iodide absorption continues to rise at as we go from visible to UV, far past 300-nm. Thus, the number of photons that were determined to strike the sensitized plate surface are not all making it to the silver layer. The AgI layer acts as a filter so that fewer photons strike the silver as we lower the wavelength, reducing the number of particles produced. If this was the case, then it is likely that the silver iodide is the culprit for the continuous drop at 302-nm, shifting it from the imaginary refractive index trend.

In addition to the shift, wavelengths 334-nm through 406-nm do not appear to follow the same trend that the imaginary refractive index does. The particles per photon curve plateaus rather than continuously rising. It is important to note that we are relying on nanoparticle production as an indicator for the occurrence of an absorption event between a specific photon and the plate. The plate is only capable of producing a certain number particles for a given area. It is likely that for wavelengths between 334-nm and 406-nm, that limit was reached, giving the appearance of a plateau. This can be confirmed by repeating the experiment with a reduced intensity of the light through the use of a neutral density filter, or reducing the duration of exposure. 
If the above issues were in fact occurring, then it is possible that particles are also being produced through the absorption in the silver substrate.

It is also important to note that the particles produced were extremely clear to see when analyzed with SEM. Furthermore, it was possible to see certain shapes clearly produced. With wavelengths $254 \mathrm{~nm}$ and $302 \mathrm{~nm}$, all the observed particles are spherical. Once we hit wavelengths $314 \mathrm{~nm}$ and $334 \mathrm{~nm}$, elongated particles are observed. Many of which appear to be comma or rod shaped, in addition to the occasional spherical shaped particle.

Since there was no control on the intensity of the excitation beam, it is uncertain as to whether this change in shape is influenced by energy, or by intensity of the beam. Wavelength $302 \mathrm{~nm}$ had a photon count of $9.4 \times 10^{27}$, similar to $334 \mathrm{~nm}$ at $1.13 \times 10^{28}$. Since they are similar to one another in photon count, the only thing that separates them is the difference in energy. And

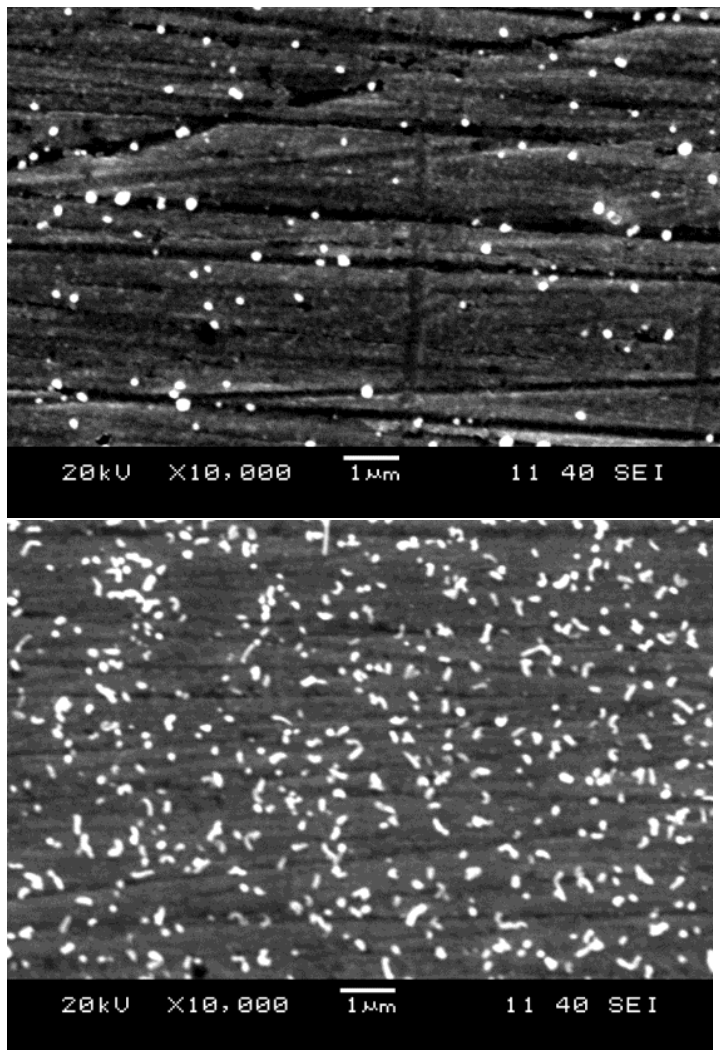

Figure 3.3: SEM images of particles produced with $302 \mathrm{~nm}$ (top) and 334nm (bottom)

since 302 produced no comma or rod shaped

particles, it is reasonable to assume that it is the energy of the light that influenced this change in shape rather than the intensity. Figure 3.3 displays the SEM images of 302nm and 334nm

\subsection{Excitation Study (No development)}


With this study, wavelengths $447 \mathrm{~nm}$

through to $530 \mathrm{~nm}$ were the only wavelengths successful at producing particles. No particles were observed on $591 \mathrm{~nm}$ to $655 \mathrm{~nm}$-LEDs. In section 3.1.1, 591-nm LED and 617-nm LED were still capable of producing particles. This experiment demonstrates that high energy light is capable of both producing particles and growth.

In addition, similar to the results of section 3.1.1, LED 447-nm produced the highest number of particles of all the LEDs, with 1456 produced. The 470-nm LED, 505-nm LED and 530-nm LED produced 220, 83 and 201 particles per unit area respectively (seen in Figure 3.5). The reasons for this are likely to be due to the nature of the silver iodide absorption spectrum at this wavelength range.

It was also observed that the particles were extremely small in size in this experiment. Particles were approximately $2447 \mathrm{~nm}^{2}$ in size. With the red light development, particles averaged

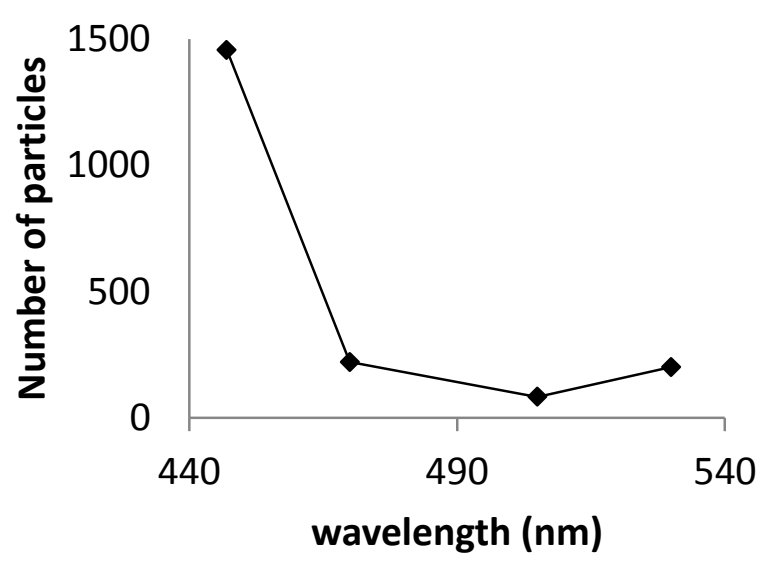

Figure 3.5: Number of particles produced with for wavelengths $447 \mathrm{~nm}, 470 \mathrm{~nm}, 505 \mathrm{~nm}$ and $530 \mathrm{~nm}$. No $655 \mathrm{~nm}$ light development was used 
at around $5000 \mathrm{~nm}^{2}$ instead. The ratio

between the number of nucleation

sites to the abundance of silver is

likely the cause of this substantial

difference in size. Figure 3.4 shows a

side by side comparison between a

developed and undeveloped image.

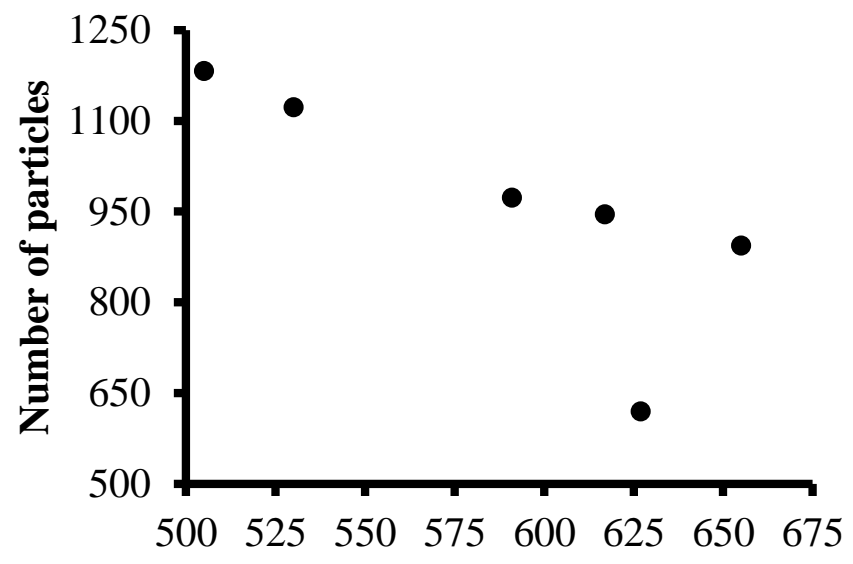

In this method, with only blue

Figure 3.6: Number of particles produced when developed with $505 \mathrm{~nm}, 530 \mathrm{~nm}, 591 \mathrm{~nm} 617 \mathrm{~nm} 627 \mathrm{~nm}$ and $655 \mathrm{~nm}$

light shining for 4 minutes,

Wavelength (nm)

nucleation sites were continuously

being produced, while the previous method only had 10 seconds of exposure. This results in significantly more nucleation sites. With more nucleation sites available, that means that for every area, there would be fewer silver atoms available per nucleation site for growth, resulting in smaller particles.

\subsection{Development Study}

Much like the excitation study, the size and number of particles will be examined to determine the production properties of each of the LEDs during the development step. The LEDs used were 505-nm, 530-nm, 591-nm, 617-nm, 627-nm, and 655-nm. All LED intensities were adjusted to match one another in photon production.

The 530-nm LED produced the largest number of particles, and the 627-nm LED produced the smallest number. Aside from LED 627-nm, the graph shows a linear decrease in particle count as the wavelength increases (Figure 3.6). 
With regards to particle size, it

was observed that LED 530-nm

produced the largest particles of all

LEDs, with a size of approximately

81nm. LED 591-nm produced the

smallest particles of approximately

$68 \mathrm{~nm}$. The rest of the LEDs produce

particles of around 70nm. Looking at the

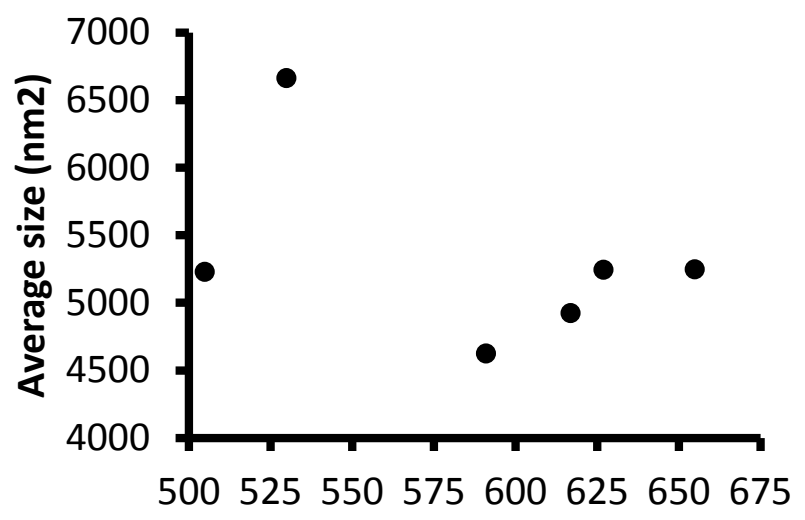

Wavelength (nm)

Figure 3.7: Average particle size produced by development with $505 \mathrm{~nm}, 530 \mathrm{~nm}, 591 \mathrm{~nm}, 617 \mathrm{~nm}, 627 \mathrm{~nm}$, and $655 \mathrm{~nm}$.

graph of particle size vs wavelength in

Figure 3.7, the particle size appears to be somewhat invariable. There is no substantial difference in size difference between each LED, with the possible exception of 530nm. This indicates that there is no correlation between particle size and wavelength, meaning the rate of growth does not depend on the wavelength of the development step within the range tested.

\subsection{Iodine Study}

\subsubsection{ICP Analysis of silver iodide maturation}

This study was done to determine whether or not the layer of silver iodide was increasing over time, given that the iodine maturation does exist. Figure 3.8 displays a graph of all plates and slices analyzed for silver iodide, to determine whether age plays a role in the particle formation. All expected AgI concentration values have been normalized with respect to the affected area of the plate. All the plates have displayed a consistent result in the quantity of silver iodide, except for plate 2 on day 3 , where the concentration of that plate is about double that of plates 1 and 3. It was also observed that the flask containing the silver iodide from plate 2 was 


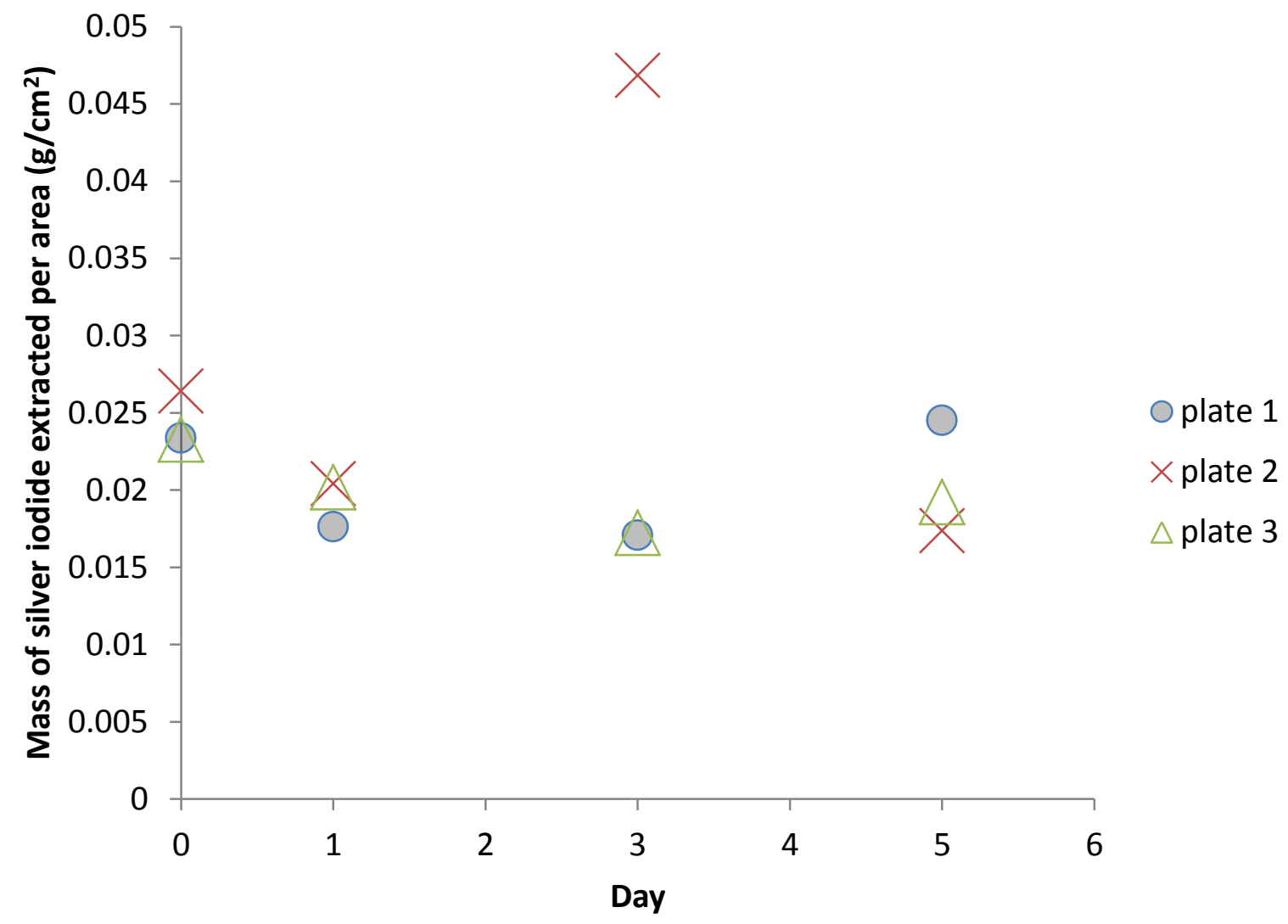

Figure 3.8: Maturation study of the silver iodide.

clear with a faint and pale yellow colour, while the rest of the 11 flasks were clear and colourless. It is unclear as to how flask 2 contains significantly higher concentrations of silver iodide compared to the rest of the flasks, but it is reasonable to conclude that it is an outlier.

With 0 days for maturation, the silver iodide extracted from the plate averaged at about $0.138 \mathrm{~g} / \mathrm{cm}^{2}$, this was the highest value of all time periods. For days 1,3 and 5 the observed concentration averaged at $0.110 \mathrm{~g} / \mathrm{cm}^{2}, 0.153 \mathrm{~g} / \mathrm{cm}^{2}$ and $.115 \mathrm{~g} / \mathrm{cm}^{2}$ respectively.

It is hypothesized that the change in the image quality due to the maturation of the plate is caused by a change in the thickness of the silver iodide layer. Since iodine is capable of adhering to silver iodide, we hypothesised that over time some of the adhered iodine would 
diffuse through the silver iodide layer, makes its way down to the bare silver substrate, and react to form additional silver iodide. This formation of new silver iodide may modify the thickness of the silver iodide layer, and impact the formation of nanoparticles. However, looking at Figure 3.8 , a decrease in the mass of silver iodide per $\mathrm{cm}^{2}$ is observed, rather than an increase.

A t-test calculation was performed on the 0 day and 1 data, to determine if the difference between them was significant enough to be caused by factors other than random error. The $\mathrm{t}$ value was determined to be 3.621 . With this t value, there is a 0.0112 probability that the difference between the data is cause by random error, and that there is no significant difference between them. Since this value is far below 0.05 (a 95\% confidence interval), it is safe to reject the null hypothesis. The difference between the data is statistically significant enough to be cause by factors other than random error.

Since the quantity of silver iodide is decreasing rather than increasing, our hypothesis on the effect of maturation on the quality of the nanoparticles is rejected. It is unclear as to why this change occurs, but it is linked to affecting the features of the produced particles.

Furthermore, a t-test was done on the data between day 1 and day 5, and day 0 and 5, to determine which data happen to be closer to day 5. The data between day 1 and 5 have the higher probability of being similar to one another, with a 0.349 probability. This indicates that the difference in data between those two days is not significant, and likely caused by random error. The probability between day 0 and 5 was only 0.0846 .

\subsubsection{SEM analysis of silver iodide maturation}

The study of time periods of minutes, hours and days for maturation revealed that there is an increase in the number of particles produced. For the minutes plate, $0,11,22$, and 33 minutes 
were explored. The number of particles produced increased linearly with the passage of time, as seen in Figure 3.9. In the hours study, the number of particles produced also increases linearly with time between 0 hours to 2 hours.

The pattern with the days study however, was different. For the first 24 hours, an increase was observed, from 930 particles on day 0 to 1502 particles on day 1. Day 3 has produced 1499 particles, no noticeable change when compared to day 1 . After 5 days, however, there was a significant decrease in particle count down to 955 particles, similar to the particle count on day 0.

Based on this experiment, it is apparent that there is in fact an increase in the number of particles over time. For the first two hours, this increase appears to be linear in nature. Since there is no change in particle count between days 1 and 3 , the effects of silver iodide maturation on particle production plateaus somewhere between 2 hours and 24 hours. If a high number of particles per unit area is desired, it is advised to allow the silver iodide to age for 24 hours prior to particle production, and not go beyond 3 days since the counts decreased in day 5 . The mechanism and factors that influence this change in particle count are still not understood.

The trend that is observed with the effects of maturation on particle production does follow a similar trend to maturations effect on amount of silver iodide. Between day 0 and day one, the increase in particle production is accompanied by a decrease in silver iodide quantity. Between day 1 and day 3, there was no change in particle production, nor was there any change in the silver iodide quantity. This trend would suggest that there is a link between the particle production and the quantity of silver iodide. This trend deviates once we reach day 5 , where the number of particles produced decreases significantly, comparable to day 0 . The silver iodide magnitude experiment, on the other hand, suggest that the amount if AgI present on day 5 is 

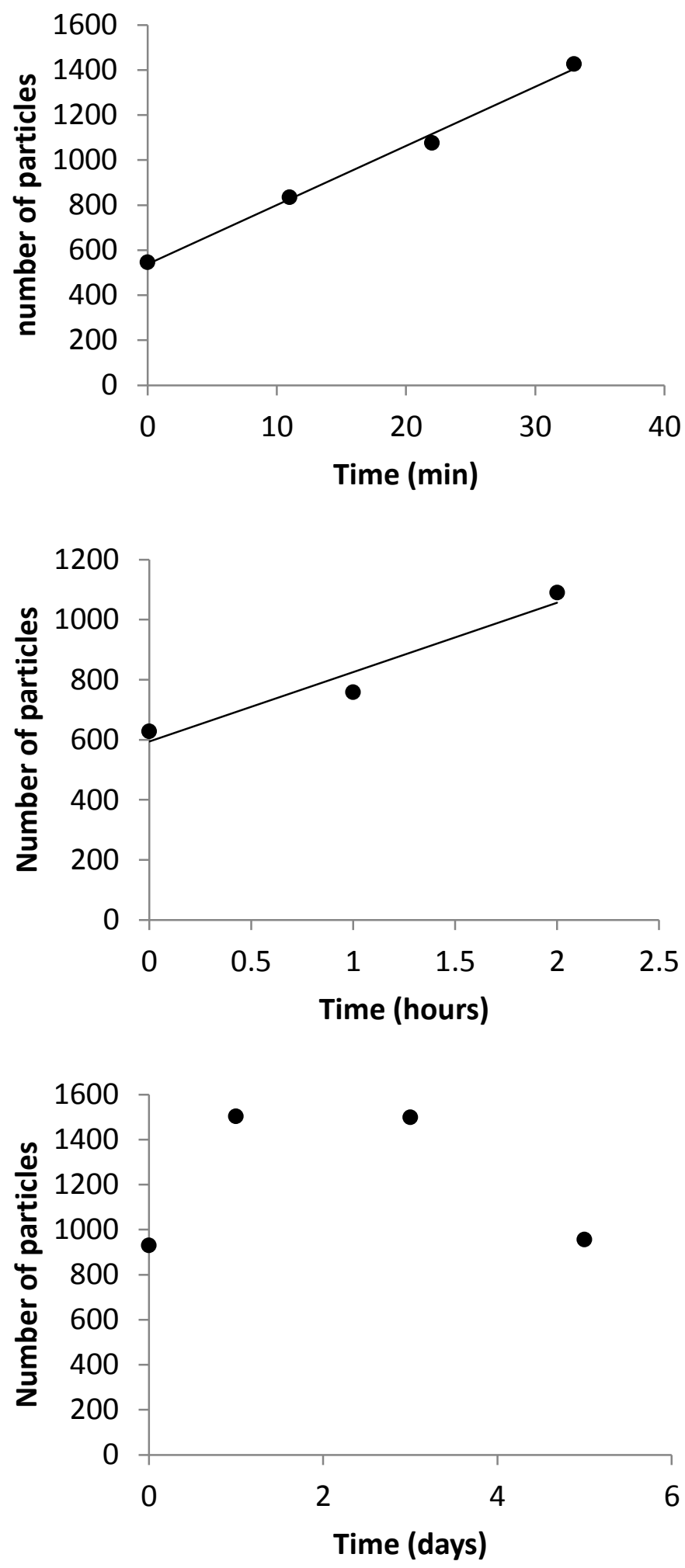

Figure 3.9: Maturation of the iodide layer. Changes in the iodide layer during the first day after iodine exposure result in enhanced particle formation. 
similar to days 1 and 3, as opposed to day 0, suggesting no change. This conclusion was further reinforced with the t-test, giving a probability of 0.349 that day 5 is comparable to day 1 . The cause of deviation in the trend is unclear at this point.

\subsection{Particle Dispersity}

While the program ImageJ did provide data about the overall SEM image, it also recorded information individual data for each particle detected. This made it possible to create a histogram for all the data, in addition to determining the polydispersity index (PDI).

For each of the histograms, the values were listed based on the mass of the particles. The bin values were calculated based on the minima of the mass, and incrementing by 10 times the

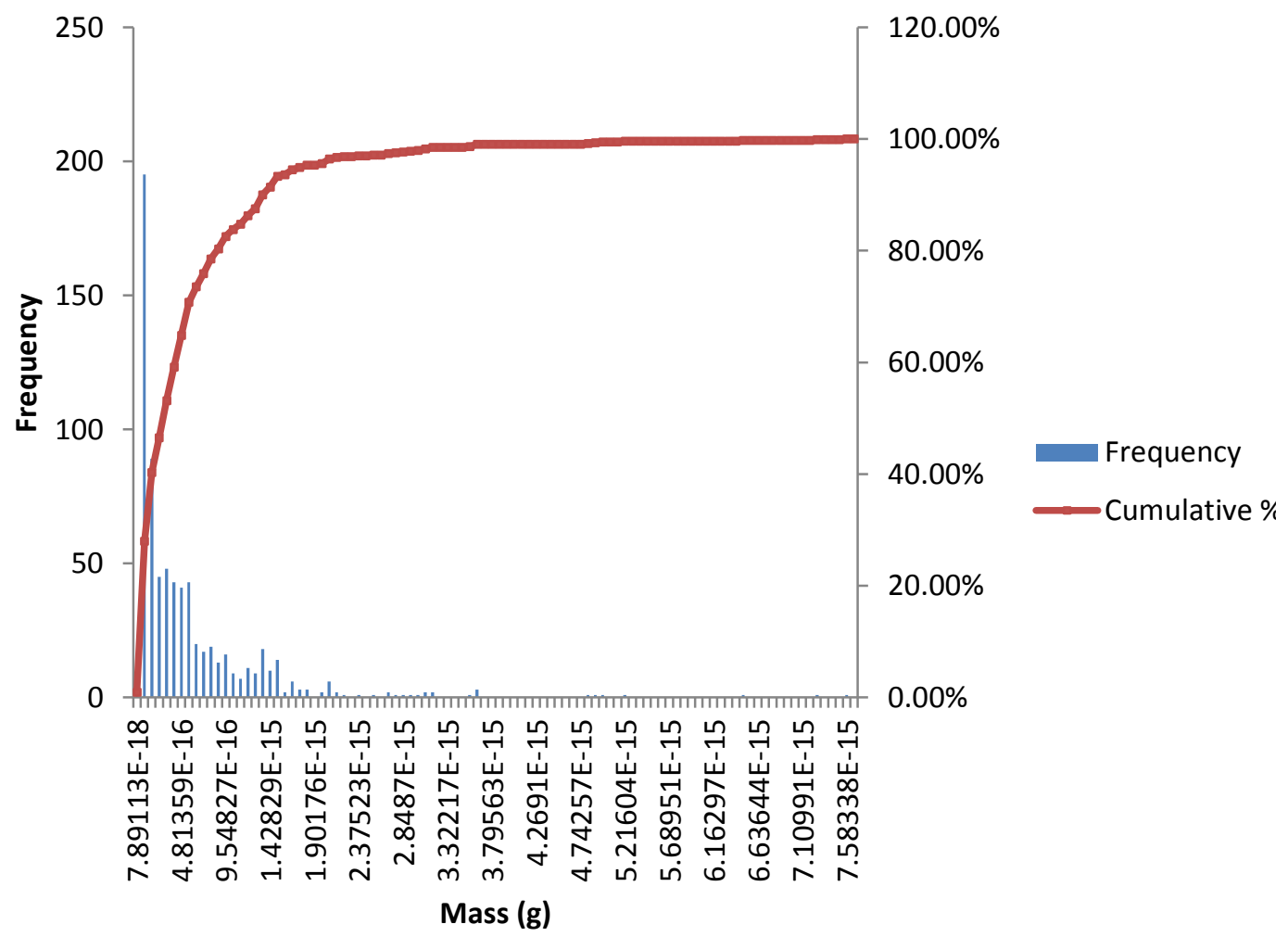

Figure 3.10: Histogram of 447nm LED exposure, developed with 655nm LED. 
minima. The mass was

calculated using the area of

the particle and density of

silver, under the assumption

that the particles are perfectly

spherical in shape. With all

the collected data, the

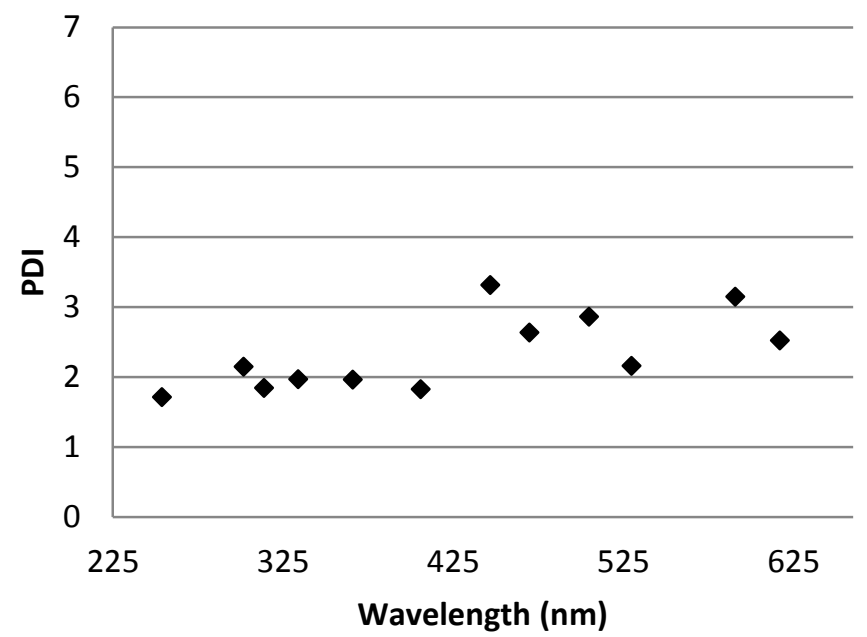

histogram distribution is

positively skewed, nearly

Figure 3.11: PDI value for the developed excitation study for both UV and visible.

$70 \%$ of the particles are present within the first few bins. The mass of the particles would range between E-18 and E-16 grams, as seen in Figure 3.10.

The PDI did vary slightly from sample to sample, but the variation is more significant when comparing one method to another.

For the visible excitation study, the 530nm LED had the lowest PDI of 2.15, and highest at 3.31 for $447 \mathrm{~nm}$. The UV side

of the experiment however,

produced much lower values,

the highest being 2.14 for

$302 \mathrm{~nm}$, and 1.71 for $254 \mathrm{~nm}$

(Figure 3.11). The PDI values

do appear to be quite scattered

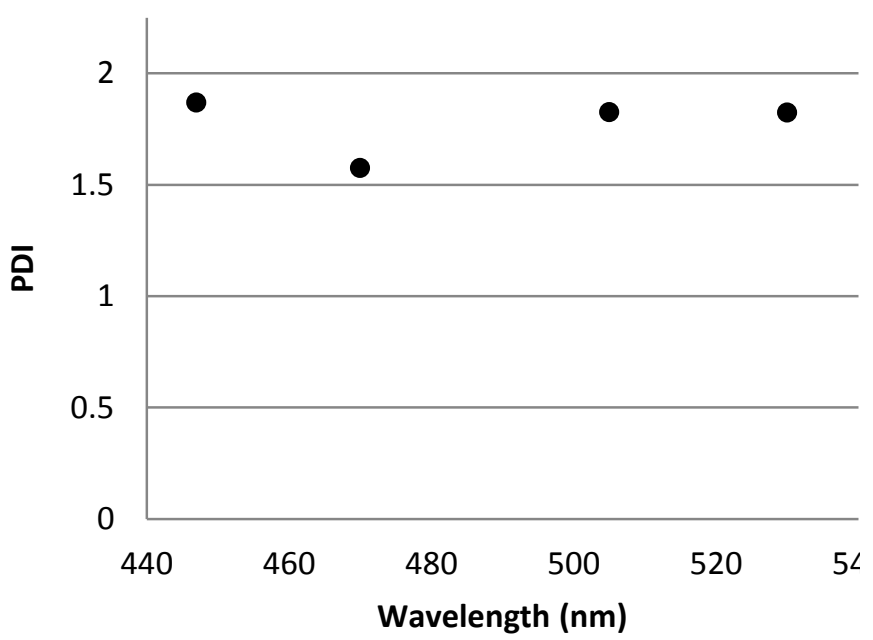

for the visible region, and

Figure 3.12: PDI value for excitation without development. 
narrow for the UV. This may indicate that the dispersity of the particles might be wavelength dependent. It is also possible that the difference could be caused by the difference in the types of sources, an intense LED, versus a mercury arc lamp whose intensity was weakened after putting it through a monochrometer.

The excitation study with no development on the other hand produced fairly small PDI values, averaging at approximately 1.77, while development had an average of 2.77 . The sizes of the particles were also extremely small when compared to the excitation with development study (Figure 3.12). It is reasonable to conclude that the difference in distribution between those two experiments is caused by the presence of the development step, which is a 10 minutes exposure to red light. That alone could account for the size and distribution of the particles. 


\section{Future work}

In these experiments, much has been learned about the Becquerel method in terms of nanoparticle formation. This ancient, yet classical method of photography may soon be used as a novel and simple method to form silver nanoparticles. However, there is still much to learn.

With regards to the excitation study, 6 LEDs were used to study the visible spectrum, and determine wavelengths effective at producing particles. Only one LED was successful at effectively producing nanoparticles, 447-nm. There is a gap between 447-nm and 470-nm. Narrowing that gap to determine more closely at which energy incident light becomes ineffective at particle production would be useful. The results would help reinforce the proposed mechanism of absorption through silver iodide, improving our understanding of the process

In addition, the UV spectrum experiment should also be repeated, with narrower energy gaps between each of the points. In addition, the excitation intensity should be reduced to determine if the curve plateau at 334-nm through 406-nm would produce a curve similar to the imaginary refractive index. This would once again reinforce the mechanisms proposed earlier.

Furthermore, creating silver iodide films on other substrates should be investigated. If it is possible to create films on other substrates, it may help reduce the final price of the plates. Again, the results of this experiment could reinforce or reject the mechanisms proposed.

The development study only looked at light development between 505-nm and 655-nm. A difference in average particle size was observed between each of the LEDs. This range could be widened further to infrared to determine the kinds of particle sizes we can create. 
The iodine study has revealed that we were successful at creating fairly reproducible silver iodide films. This reproducibility can be further improved through controlling the pressure of the environment, a factor that we didn't have the means of controlling. With regards to the silver iodide maturation, the $5^{\text {th }}$ day revealed a decrease in the production of nanoparticles, which went against our theory and its link with silver iodide mass. The experiment should be repeated, and extend the number of days. Not only to confirm our initial finds, but to determine if this pattern continues beyond 5 days.

The research that was done on the particles in this study was conducted with SERS application in mind. Experiments should be conducted to determine its use in this application. In addition, since SERS is capable of detecting a single molecule in a given area, experiments should be done to determine if it is possible with the Becquerel plate, and determine what conditions are required to achieve such a level of detection. 


\section{Conclusion}

The experiments done in this study were conducted to enhance our understanding of the silver nanoparticle production, and determine the factors that control the characteristics of the particles. With the excitation study, it was determined that wavelength 447-nm was the only wavelength in the visible range that was successful at producing nanoparticles effectively. This happens to also be the beginning of absorption spectra of silver iodide, which suggests that the production mechanism occurs in the silver iodide layer. It was also possible for UV to produce particles. All mercury arc lamp peaks between 406-nm and 254-nm were successful. Their absorption happens to be similar to the absorption of the silver plate. This may suggest that the plate may also be involved in the mechanism of the particle production. The development study revealed that all wavelengths between $505-\mathrm{nm}$ to $655-\mathrm{nm}$ were successful at developing the image; development is not limited to red light alone. In addition, the average particle size can also be controlled by the wavelength, with 505 providing largest average size, and $655 \mathrm{~nm}$ producing the smallest. The silver iodide study revealed that maturation effect of silver iodide does exist. It was determined that maturation causes a diminishment in silver iodide layer, in addition to an increase in the number of particles produced. A link between the two may be present, and can be used as another level of control. 


\section{References}

(1) Neouze, M.-A. J. Mater. Sci. 2013, 48, 7321-7349.

(2) Nair, L. S.; Laurencin, C. T. J. Biomed. Nanotechnol. 2007, 3, 301-316.

(3) Yu, S.; Yin, Y.; Liu, J. Environ. Sci. Process. Impacts 2013, 15, 78.

(4) Krutyakov, Y. a; Kudrinskiy, a a; Olenin, a Y.; Lisichkin, G. V. Russ. Chem. Rev. 2008, 77, 233-257.

(5) Yu, S.; Yin, Y.; Liu, J. Environ. Sci. Process. Impacts 2013, 15, 78.

(6) Silvestry-Rodriguez, N.; Sicairos-Ruelas, E.; Gerba, C.; Bright, K. Rev. Environ. Contam. Toxicol. 2007, 191, 23-45.

(7) Parashar, U. K.; Kumar, V.; Bera, T.; Saxena, P. S.; Nath, G.; Srivastava, S. K.; Giri, R.; Srivastava, A. Nanotechnology 2011, 22, 415104.

(8) Galdiero, S.; Falanga, A.; Vitiello, M.; Cantisani, M.; Marra, V.; Galdiero, M. Molecules 2011, 16, 8894-8918.

(9) Tian, J.; Wong, K. K. Y.; Ho, C.-M.; Lok, C.-N.; Yu, W.-Y.; Che, C.-M.; Chiu, J.-F.; Tam, P. K. H. ChemMedChem 2007, 2, 129-136.

(10) Rycenga, M.; Camargo, P. H. C.; Li, W.; Moran, C. H.; Xia, Y. J. Phys. Chem. Lett. 2010, $1,696-703$.

(11) Sharma, B.; Frontiera, R. R.; Henry, A.; Ringe, E.; Duyne, R. P. Van. Mater. Today 2012, $15,16-25$.

(12) Fleischmann, M. 1974, 26, 2-5.

(13) Moskovits, M. J. Raman Spectrosc. 2005, 36, 485-496.

(14) Van Hoonacker, A.; Englebienne, P. Curr. Nanosci. 2006, 2, 359-371.

(15) Jacob, J. a.; Kapoor, S.; Biswas, N.; Mukherjee, T. Colloids Surfaces A Physicochem. Eng. Asp. 2007, 301, 329-334.

(16) Velikov, K. P.; Zegers, G. E.; Blaaderen, A. Van. 2003, 1384-1389.

(17) Wang, H.; Qiao, X.; Chen, J.; Wang, X.; Ding, S. Mater. Chem. Phys. 2005, 94, 449-453. 
(18) Xu, G.; Qiao, X.; Qiu, X.; Chen, J. Colloids Surfaces A Physicochem. Eng. Asp. 2008, $320,222-226$.

(19) Li, T.; Park, H. G.; Choi, S.-H. Mater. Chem. Phys. 2007, 105, 325-330.

(20) Liu, F.-K.; Hsu, Y.-C.; Tsai, M.-H.; Chu, T.-C. Mater. Lett. 2007, 61, 2402-2405.

(21) Kshirsagar, P.; Sangaru, S. S.; Malvindi, M. A.; Martiradonna, L.; Cingolani, R.; Pompa, P. P. Colloids Surfaces A Physicochem. Eng. Asp. 2011, 392, 264-270.

(22) Stamplecoskie, K. G.; Scaiano, J. C. J. Am. Chem. Soc. 2010, 132, 1825-1827.

(23) Tsuji, T.; Watanabe, N.; Tsuji, M. Appl. Surf. Sci. 2003, 211, 189-193.

(24) Mafuné,, F.; Kohno, J.; Takeda, Y.; Kondow, T.; Sawabe, H. J. Phys. Chem. B 2000, 104, 9111-9117.

(25) Jiang, H.; Moon, K.; Zhang, Z.; Pothukuchi, S.; Wong, C. P. J. Nanoparticle Res. 2006, 8, $117-124$.

(26) Salkar, R. a.; Jeevanandam, P.; Aruna, S. T.; Koltypin, Y.; Gedanken, a. J. Mater. Chem. 1999, 9, 1333-1335.

(27) Li, X.; Xu, W.; Zhang, J.; Jia, H.; Yang, B.; Zhao, B.; Li, B.; Ozaki, Y. Langmuir 2004, 20, 1298-1304.

(28) Barger, S. M.; White, W. B. The Daguerreotype, Nineteent century technology and modern science; illustrated, reprint, Ed.; Johns Hopkins University Press, 2000; p. 252.

(29) Pobboravsky, I. Study of iodized daguerreotype plates; Information Service, Graphic Arts Research Center, Rochester Institute of Technology: Rochester, NY, 1971; p. 60.

(30) Cochrane, G. J. Phys. D. Appl. Phys. 1974, 7, 748-758.

(31) Kurdyumova, R. N.; Sviridov, D. T. Optical Properties of Silver Iodide Films, 1968, 643644. 